\title{
A!
}

This is an electronic reprint of the original article.

This reprint may differ from the original in pagination and typographic detail.

Laurila, Sara; Nissinen, Jaakko

\section{Torsional Landau levels and geometric anomalies in condensed matter Weyl systems}

Published in:

Physical Review B

DOI:

10.1103/PhysRevB.102.235163

Published: 30/12/2020

Document Version

Publisher's PDF, also known as Version of record

Please cite the original version:

Laurila, S., \& Nissinen, J. (2020). Torsional Landau levels and geometric anomalies in condensed matter Weyl systems. Physical Review B, 102(23), [235163]. https://doi.org/10.1103/PhysRevB.102.235163

This material is protected by copyright and other intellectual property rights, and duplication or sale of all or part of any of the repository collections is not permitted, except that material may be duplicated by you for your research use or educational purposes in electronic or print form. You must obtain permission for any other use. Electronic or print copies may not be offered, whether for sale or otherwise to anyone who is not an authorised user. 


\title{
Torsional Landau levels and geometric anomalies in condensed matter Weyl systems
}

\author{
Sara Laurila (1) ${ }^{*}$ and Jaakko Nissinen ( $^{\dagger}$ \\ Low Temperature Laboratory, Department of Applied Physics, Aalto University, P.O. Box 15100, FI-00076 Aalto, Finland
}

(Received 26 August 2020; accepted 20 November 2020; published 30 December 2020)

\begin{abstract}
We consider the role of coordinate-dependent Fermi velocities, equivalent to effective tetrad (or vierbein) frame fields characterizing momentum space geometry, and torsional Landau levels (LLs) in condensed matter systems with low-energy Weyl quasiparticles. In contrast to their relativistic counterparts, they arise at finite momenta and with an explicit cutoff to the linear spectrum. Via the universal coupling of tetrads to momentum, they experience geometric chiral and axial anomalies with gravitational character. More precisely, at low energy, the fermions experience background fields corresponding to emergent anisotropic Riemann-Cartan and Newton-Cartan space-times, depending on the form of the low-energy dispersion. On these backgrounds, we show how torsion and the Nieh-Yan (NY) anomaly appear in condensed matter Weyl systems with an ultraviolet (UV) parameter with dimensions of momentum. The torsional NY anomaly arises from the spectral flow of torsional LLs and the linear Weyl description with a cutoff. We carefully review the torsional anomaly and spectral flow for relativistic fermions at zero momentum and contrast this with the spectral flow, nonzero torsional anomaly, and the appearance of the dimensionful UV-cutoff parameter in condensed matter systems at finite momentum. We apply this to chiral transport anomalies sensitive to the emergent tetrads in nonhomogeneous chiral superconductors, superfluids, and Weyl semimetals under elastic strain. This leads to previously overlooked suppression of anomalous density at nodes from momentum space geometry, as compared to (pseudo)gauge fields. We also briefly discuss torsion in anomalous thermal transport for nonrelativistic Weyl fermions, which arises via Luttinger's fictitious gravitational field corresponding to thermal gradients.
\end{abstract}

DOI: 10.1103/PhysRevB.102.235163

\section{INTRODUCTION}

Gapless fermionic quasiparticles with linear spectrum protected by topology arise in many condensed matter systems in three dimensions [1-5]. In particular, accidental crossings of two inversion $(P)$ or time-reversal $(T)$ breaking bands at the Fermi energy lead to stable quasirelativistic particles with low-energy dispersion analogous to relativistic Weyl fermions [6,7]. Fourfold-degenerate crossings with Diractype low-energy excitations occur for combined $P, T$ (and/or other similar protecting) symmetries [8,9]. Similarly, in chiral superconductors and superfluids with gap nodes, MajoranaWeyl excitations arise at low energy [3,10-14].

By a very general theorem from topology [4], the lowenergy linear theory near the three-dimensional Fermi point node takes universally the ( $\gamma$-matrix) form of a quasirelativistic Weyl/Dirac spectrum, with the precise form of the metric and other background fields depending on the microscopic details. It is then of interest to study the detailed form of this emergent Dirac operator with an explicit cutoff and compare to fundamental, Lorentz-invariant fermions. Following this logic, the concept of so-called momentum space pseudogauge fields [3,15-26] and "emergent" space-time [3,11-15,27-45] in nonrelativistic condensed matter systems has emerged, where the low-energy fermions can experience background

\footnotetext{
*sara.laurila@helsinki.fi

†jaakko.nissinen@aalto.fi
}

fields of various physical origins, similar to what appears for spin- $\frac{1}{2}$ (or even higher spin) fermions on curved space-times in general relativity or its nonrelativistic generalizations with coordinate invariance.

In the low-energy quasilinear theory, the local Fermi velocities form emergent tetrads (or spatial dreibein) frame fields which determine the geometry of the conical dispersion. The tetrads, and their field strength torsion, couple to the quasiparticle momentum effectively as in gravity. Moreover, the appearance of such background fields in condensed matter Weyl systems is built in in the low-energy theory, thus opening the possibility of simulating Riemann-Cartan (or NewtonCartan) space-times for the low-energy fermions. The effects of such fields in nonrelativistic systems appearing at finite density $\mu_{F}$ and Fermi momentum $p_{F}$ are expected to be very different from their relativistic counterparts appearing at $p=\mu=0$. Notably, the system at finite Fermi or crystal momentum is charged under the geometric background fields [15,30,38,42-45].

In three spatial dimensions Weyl fermions experience chiral anomalies [46]. Here, the anomalous chiral symmetries leading to axial anomalies in the system [38,47] correspond to translational symmetries in momentum space. For relevant condensed matter considerations of the chiral anomaly, see, e.g., [3,9,11,48-63]. In the case of nontrivial background torsion, the so-called chiral gravitational Nieh-Yan (NY) anomaly can appear $[64,65]$. In contrast to the axial anomaly with gauge fields, this anomaly depends on a nonuniversal high-energy or ultraviolet (UV) cutoff parameter $\Lambda$, 
with canonical dimensions of momentum. While the status of the torsional anomaly contribution in relativistic systems has been debated for long [66-72], the appearance of this term in nonrelativistic condensed matter systems with explicit an UV cutoff to the Weyl physics is a priori plausible $[38,44]$. In this paper, following [38], we point out that geometric (gravitational) contributions in the chiral anomaly, second order in gradients, are expected in generic nonhomogeneous condensed matter Weyl systems due to inhomogeneous torsional deformations. Our central result is simple: from the torsional Landau levels (LLs), we compute the adiabatic spectral flow. Due to momentum playing the role of effective charge, the LL density of states is momentum dependent and leads to a momentum dependence in the spectral flow. For the anomaly, this implies the nonuniversal UV cutoff $\Lambda$, arising from the breaking of the Weyl approximation and no well-defined chirality outside the low-energy spectrum close to the nodes. We apply this to anomalous transport in chiral superfluids and superconductors $[3,38,54]$ and semimetals under elastic strain $[15,27,28,30]$. Although these systems have been shown to have nonhomogeneous tetrads under deformations, the gravitational (geometric) nature of the anomaly and the suppression [38] from the small "low-energy UV scale" $\Lambda$ in momentum space in the latter systems seems to have been ignored in the literature, to the best of our knowledge. The suppression is expected to be experimentally significant.

\section{Previous work and detailed organization of the paper}

The torsional LLs appeared implicitly already in Refs. [10,51] and more recently in topological semimetals in [44] in comparison with Pauli-Villars regularization of Lorentz-invariant fermions. Such a relativistic regularization scheme is at best only an approximation in condensed matter systems since the linear Weyl regime applies to low energies with an explicit cutoff scale. This linear regime can be anisotropic and, furthermore, is continuously connected with the nonrelativistic regime with quadratic dispersion. In addition, as discussed in this paper, the role of the spectral flow is drastically altered by the finite node momentum as compared to relativistic fermions.

Aspects of gravitational anomalies in condensed matter have been considered in, e.g., Refs. [38,44,58,59,63,73-80] including Weyl and Dirac fermions in superfluids, superconductors, and semimetals. The dimensional hierarchy and descent relations of the torsional anomaly were recently analyzed in Ref. [79] from a Hamiltonian perspective in a relativistic model. Nevertheless, it seems that any explicit value of the cutoff parameter $\Lambda$ has not been discussed in detail, except in the recent papers $[38,81]$ by one of the present authors. In the simplest possible terms, the nonuniversal UV scale originates from the regime of validity of the Taylor expansion and well-defined chirality close to the node. Experimentally, this is a low-energy scale in the system [38]. Generalizing this observation to arbitrary Weyl fermion systems, we verify that the NY anomaly nonuniversally probes the chiral spectrum and transport, and is well defined only upon a UV completion in some left-right asymmetric way, as required by global consistency and symmetries. Indeed, at face value, the spectrum and spectral flow can be terminated in a multitude of inequivalent ways. If the system is anisotropic, the interplay of different scales in the system becomes essential.

Our geometric results clarify the role of pseudogauge fields with momentum-dependent axial charge from, e.g., elastic deformations [15,27,28]. Evident in the geometric framework for the axial anomaly presented here, it is incorrect to assume the universal U(1) axial anomaly for such gauge fields since the effective momentum space description has a finite regime of validity leading to a suppression of that magnitude from the LL density of states. The results coincide only if the Weyl approximation is valid in the whole spectrum, which is impossible in condensed matter systems; to our knowledge this has been overlooked thus far in the literature.

Related to the scale dependence in the torsional anomaly, the UV scale can be supplemented by an infrared (IR) temperature scale of universal thermal fluctuations with some caveats. The thermal torsional anomaly and the associated currents were recently considered in Ref. [81]. Contributions from torsion at finite temperatures were further discussed in [82-87] for relativistic fermions at $p=0$. The closely related role of torsion and viscoelastic thermal transport has been also studied, e.g. in [88-91]. Here, we mostly focus on the nonuniversal UV contribution at zero temperature. For completeness, we comment on thermal effects by nonzero temperature gradients, which point to still new types of anisotropic torsional anomalies terms not present in systems with Lorentz invariance.

The rest of this paper is organized as follows. Section II discusses the low-energy Weyl Hamiltonian, the associated geometry, and the torsional anomaly in condensed matter systems. The following Sec. III reviews the torsional LLs and spectral flow argument for the anomaly, followed by the extension to finite node momentum and the comparison with the anomaly for U(1) gauge fields (see also Appendices $\mathrm{A}$ and $\mathrm{C}$ ). In Sec. IV we apply the torsional anomaly in chiral superfluids and superconductors, where it can be matched with experiment [38,53,54,81]. This is followed by (re)considering the torsional anomaly in $T$-breaking strained semimetals in Sec. V. We also briefly discuss the role of torsion in the presence of thermal gradients in Sec. VI. We conclude with an outlook of our results in Sec. VII. In Appendix A we review the anomaly and spectral flow for U(1) gauge fields. Emergent space-times corresponding to consistent background geometries of low-energy Weyl fermions with nonrelativistic dispersions are discussed in Appendix B. A detailed comparison of previous results using pseudogauge fields is given in Appendix C. The Appendices can be skipped by readers interested in the torsional LL results and the anomaly only.

\section{WEYL FERMIONS, GEOMETRY, AND CHIRAL ANOMALIES IN CONDENSED MATTER SYSTEMS}

\section{A. Weyl fermions in condensed matter}

We consider a fermionic system with broken time-reversal $(T)$ or inversion $(P)$ symmetry. In the vicinity of a generic degenerate crossing at $\mathbf{p}_{W}$, ignoring all other bands, the $2 \times 2$ 
Hamiltonian is $H=\sigma^{a} H_{a}$ in terms of the unit and Pauli matrices $\sigma^{a}, a=0,1,2,3$. This leads to the expansion

$$
H(\mathbf{p})=\sigma^{a} e_{a}^{i}\left(p-p_{W}\right)_{i}+\cdots,
$$

where the matrix coeffiecients with dimensions of velocity

$$
e_{a}^{i}=\left.\frac{\partial H_{a}}{\partial p_{i}}\right|_{p=p_{W}}
$$

couple the momentum and (pseudo)spin in (1) as effective tetrad (or dreibein) frame fields [3,92]. $\mathbf{p}_{W}$ is the location of the Weyl point. Due to at least broken $P$ or $T$, the shift vector

$$
p_{W, \mu}=\left(\mu_{W}, \mathbf{p}_{W}\right)
$$

is necessarily nonzero for the existence of the Weyl point. We will focus on the $T$-breaking case with two nodes of opposite chirality at $\pm \mathbf{p}_{W}$. The effects from nonzero $\mu_{W}$ can be incorporated independently and we set it to zero. The existence of the Weyl node degeneracy is protected by topology in a finite parameter region since there are three parameters and three constraints $[3,4,6,7]$. The expansion (1) is, of course, valid for $\left|\mathbf{p}-\mathbf{p}_{W}\right| \ll p_{W}$ since the remainder is (typically) of the order of $\left|\mathbf{p}-\mathbf{p}_{W}\right|^{2}$. This provides an explicit cutoff for the linear Weyl regime that is, nevertheless, continuously connected with the nonrelativistic dispersion at higher energies.

After rotations and scalings, we define $v_{F} \tilde{p}_{a}=e_{a}^{i} p_{i}$ with uniform Fermi velocity $v_{F}$ playing the role of the speed of light. The Hamiltonian becomes the right- or left-handed relativistic Weyl Hamiltonian, at Fermi momentum $\tilde{p}_{W}$,

$$
\tilde{H}(\mathbf{p})=\chi v_{F} \sigma^{a}\left(\tilde{p}-\tilde{p}_{W}\right)_{a}=\chi v_{F} \sigma^{a} \tilde{q}_{a},
$$

where $\chi= \pm 1=$ sgne is the chirality of $e=\operatorname{det} e_{a}^{i}$, defined as the direction of (pseudo)spin with respect to the propagation momentum. The band energies are $E=\tilde{q}_{0} \pm|\tilde{\mathbf{q}}|$, where $\tilde{q}_{a}=$ $\left(\tilde{p}-\tilde{p}_{W}\right)_{a}, \tilde{q}_{0}$ being a constant energy shift.

The role of the tetrad coefficients $e_{a}^{i}$ is simply to determine the (anisotropic) Fermi velocities of the conical dispersion $\omega^{2}=-g^{i j} q_{i} q_{j}$ via the (inverse) metric $[3,92]$

$$
g^{i j}=-\sum_{a, b=0,1,2,3} e_{a}^{i} e_{b}^{j} \delta^{a b} \equiv-e_{a}^{i} e_{b}^{j} \delta^{a b},
$$

where the Einstein summation convention for repeated latin and greek indices will be henceforth assumed. The spatial tetrad $e_{a}^{i}$ is extended to a nondegenerate matrix $e_{a}^{\mu}$ by considering the operator $\sigma^{a} e_{a}^{\mu} i \partial_{\mu}=i \partial_{t}-H(\mathbf{p})$ with $\mu=t, x, y, z$. The tetrad matrix and its inverse give the relation between the metrics in coordinate and orthogonal bases,

$$
g_{\mu \nu}=e_{\mu}^{a} e_{\nu}^{b} \eta_{a b}, \quad \eta_{a b}=e_{a}^{\mu} e_{b}^{\nu} g_{\mu \nu},
$$

where $\eta_{a b}=\operatorname{diag}(1,-1,-1,-1)$ is the Lorentz metric. In particular, the coefficient $e_{0}^{\mu}=\left\{1, v^{i}\right\}$ has nontrivial offdiagonal components $v^{i}$ in type-II Weyl semimetals and in superfluids and superconductors with superflow. The case with nonzero spatial $e_{a}^{t}, a=1,2,3$, was considered in [33]. The tetrad coefficients break different symmetries: While the timelike components transform like velocities, the spacelike tetrads transform like gauge potentials corresponding to axial magnetic and electric fields. The Hamiltonian (1) is usually analyzed for translationally invariant systems but it remains valid for weak deformations in coordinate space. This can be seen in any consistent gradient expansion scheme, e.g., the semiclassical gradient expansion of the Bogoliubov-de Gennes (BdG) Hamiltonian for superconductors and superfluids, or the Schrieffer-Wolff transformation for Bloch Hamiltonians $[34,36]$.

\section{B. Geometry and chiral anomalies}

We conclude that the Hamiltonian (1) has striking similarity to relativistic fermions coupled to nontrivial background geometry or gravity, albeit with some important caveats related to the nonrelativistic condensed matter system. More precisely, if we consider the low-energy Weyl fermion $\Psi_{W}$ in terms of the original excitations $\Psi$, we see

$$
\Psi(\mathbf{x}, t)=e^{ \pm i \mathbf{p}_{W} \cdot \mathbf{x}} \Psi_{W}(\mathbf{x}, t),
$$

which, however, corresponds to the anomalous (chiral) rotations in the system, thus making the finite node momentum $\mathbf{p}_{W}$ very important [38].

The chiral anomaly pertains to the breaking of the classical symmetry (7) of the massless Weyl fermions. For a pair of fermions with opposite chirality $\chi= \pm 1$ and $\mathrm{U}(1)$ gauge field $A_{\mu}$ with electric charge $q$, (1) becomes

$$
i \partial_{t}-H_{\chi}(\hat{\mathbf{p}})=\sigma_{\chi}^{a} e_{a}^{\mu}(x)\left[-i \partial_{\mu}-q A_{\mu}(x)\right]
$$

with $\sigma_{\chi=+1}^{a}=\sigma^{a}$ and $\sigma_{\chi=-1}=\bar{\sigma}^{a} \equiv\left(\mathbf{1},-\sigma^{i}\right)$. The shifted momentum is $-i \partial_{i} \sim \mathbf{q}=\mathbf{p} \mp \mathbf{p}_{W}$ from (7). The quantum anomaly is the nonconservation of the axial current $j_{5}^{\mu} \equiv$ $j_{+}^{\mu}-j_{-}^{\mu}$ for the two nodes (see Appendices A and B 3). The ensuing relativistic anomaly of (8) with $\mathrm{U}(1)$ gauge field and torsional tetrads is $[1,3,38,43,44,46,56,68,78,79,93]$

$$
\partial_{\mu}\left(e j_{5}^{\mu}\right)=\frac{\epsilon^{\mu \nu \lambda \rho}}{16 \pi^{2}}\left(q^{2} F_{\mu \nu} F_{\lambda \rho}+\Lambda^{2} \eta_{a b} T_{\mu \nu}^{a} T_{\lambda \rho}^{b}\right)+\cdots,
$$

where the torsion $T_{\mu \nu}^{a}=\partial_{\mu} e_{\mu}^{a}-\partial_{\nu} e_{\mu}^{a}$ is a geometric field strength from the tetrads similar to the electromagnetic field $F_{\mu \nu}=\partial_{\mu} A_{\nu}-\partial_{\nu} A_{\mu}$. The ellipsis signifies neglected higherorder and mixed gauge-torsional terms [72,93] not relevant here. Loosely speaking, the difference in the anomaly terms from gauge fields and tetrads can be understood from the coefficients in (9) combined with the chiral rotation (7). The anomaly, and chiral rotation, for $\mathrm{U}(1)$ gauge fields is proportional to electric charge $q$ and all charged states contribute universally. In contrast, since the the tetrads are dimensionless (in canonical units where $v_{F}=1$ ), the torsional anomaly term from (7) must be supplemented with a dimensionful parameter $\Lambda^{2} \eta_{a b}$ with dimensions of momentum squared.

In the rest of the paper, we consider the anomaly implied by (7) and (9) in the presence of nontrivial background fields $e_{a}^{\mu}(x)$ from Eq. (2) when $\mathrm{U}(1)$ gauge fields are absent. We will focus here on $T$-breaking systems, where in the simplest case one finds two Weyl nodes of opposite chirality at $\pm \mathbf{p}_{W}$, whereas for inversion $P$-breaking systems one has at minimum four Weyl points, which are invariant under $T$ and transform nontrivially under inversion [9]. The momentum (or energy) scale $\Lambda$ is computed using LLs, and its manifestations are discussed in relativistic and nonrelativistic systems. The anomaly coefficient $\Lambda$ is related to the node momentum, the breaking of Lorentz symmetries, and chirality in the UV [68], and can be anisotropic in condensed matter systems. 
For the torsional LL problem, the nonuniversal prefactor $\Lambda$ simply results from the spectral flow with momentumdependent charge and density of states in Sec. III. See Appendix A for a review of the U(1) anomaly in terms of LL spectral flow. The relevant gravitational background geometries that arise in the low-energy theory in the form of quasirelativistic and nonrelativistic low-energy space-times are discussed in Appendix B. We also review the gravitational anomaly term from torsion in more detail in Appendix B 3 . Readers interested only in the the torsional LLs and the final expression for the anomaly can move directly to the next section.

\section{TORSIONAL ANOMALIES AND LANDAU LEVELS}

Now, we proceed to compute the torsional NY anomaly (9) in nonrelativistic systems utilizing the Landau level argument. To set the stage and remove confusions before presenting our main results, we briefly review (quasi)relativistic torsional Landau levels with linear spectrum (see, e.g., [44]). The computation of the Landau levels is close to and inspired by the spectral flow obtained in $[10,51]$ for momentum space gauge fields at $p_{W} \neq 0$ but the end result will be different. Similar considerations for Weyl fermions at $p=0$ can be found in [79,84].

The Weyl particles are governed by the effective Hamiltonian (1). Throughout the paper, we assume that the coordinate dependence of the Hamiltonian arises solely from the tetrad $e_{a}^{\mu}(x)$, while the location of the node $p_{W a}$ is assumed to be constant. Note that the coordinate node momentum $p_{W \mu} \equiv e_{\mu}^{a} p_{W a}$ can still vary and when $T_{\mu \nu}^{a} \neq 0$ there is nonzero torsion. Torsional LLs arise when, say, $\frac{1}{2} \epsilon^{i j k} T_{j k}^{3}=T_{B} \hat{\mathbf{z}}^{i}$ is constant with the other torsion components and spin connection vanishing. We discuss later in Secs. IV and V on how to make the identification between low-energy emergent gravitational fields and microscopic background fields in specific examples.

\section{A. Torsional anomaly and spectral flow at $p_{W}=0$}

The (semiclassical) tetrads $e^{a}=e_{\mu}^{a} d x^{\mu}$ and the inverse $e_{a}=e_{a}^{\mu} \partial_{\mu}$ with torsion perpendicular to the $x y$ plane are, following [10,44,51],

$$
\begin{aligned}
& e^{0}=d t, \quad e^{1}=d x, \quad e^{2}=d y, \quad e^{3}=d z-T(y) d x, \\
& e_{0}=\partial_{t}, \quad e_{1}=\partial_{x}+T(y) \partial_{z}, \quad e_{2}=\partial_{y}, \quad e_{3}=\partial_{z} .
\end{aligned}
$$

Now, we compute the spectrum of the Weyl fermions in the presence of a constant torsional magnetic field $T(y)=T_{B}^{3} y$. The corresponding metric is

$$
\begin{aligned}
g_{\mu \nu} d x^{\mu} d x^{\nu}= & \eta_{a b} e^{a} e^{b} \\
= & d t^{2}-\left[1+T(y)^{2}\right] d x^{2}-d y^{2} \\
& +2 T(y) d x d z-d z^{2} .
\end{aligned}
$$

The torsion is given as $T_{i j}^{3}=\partial_{\mu} e_{\nu}^{3}-\partial_{\nu} e_{\mu}^{3}$, i.e., with nonvanishing $T_{x y}^{3}=-\partial_{y} T(y)=T_{B}^{3}$. In differential form notation of Appendix B $3, T^{3}=d e^{3}=\frac{1}{2} T_{B}^{3} d x \wedge d y$. In analogy with the electromagnetic tensor, we will call $\frac{1}{2} e^{-1} \epsilon^{i j k} T_{j k}^{a}$ and $T_{0 i}^{a}$ torsional magnetic and electric fields, respectively.

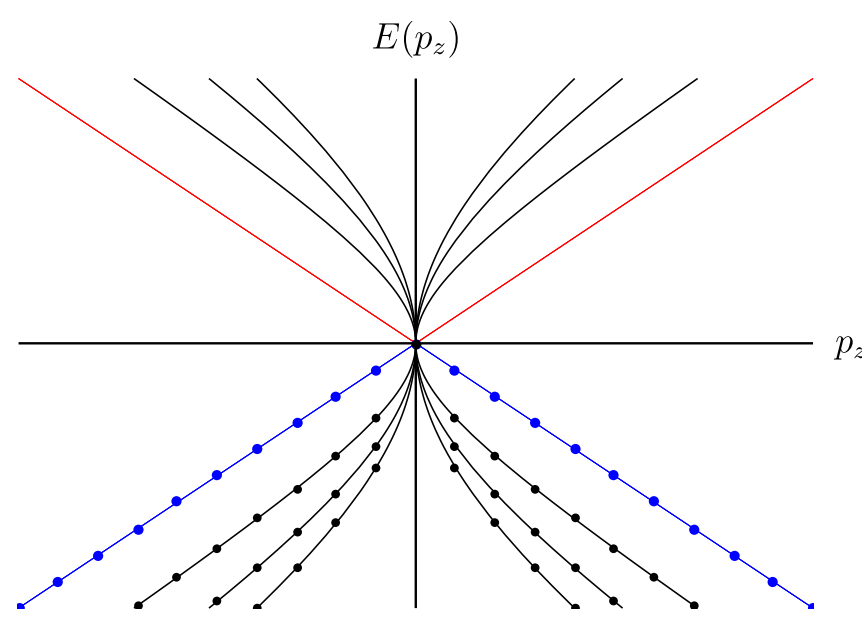

FIG. 1. Dispersion of left-handed (LLL in blue) and right-handed Weyl fermions (LLL in red) at $p_{W}=0$ under a torsional magnetic field, respectively.

The Weyl Hamiltonian couples to the nontrivial vierbein as, $\chi$ being the chirality,

$$
\begin{aligned}
H_{\chi} & =\frac{\chi}{2} \sigma^{a} e_{a}^{i} \hat{p}_{i}+\text { H.c. } \\
& =\chi\left[\begin{array}{cc}
\hat{p}_{z} & \hat{p}_{x}+\hat{p}_{z} T_{B}^{3} y-i \hat{p}_{y} \\
\hat{p}_{x}+\hat{p}_{z} T_{B}^{3} y+i \hat{p}_{y} & -\hat{p}_{z}
\end{array}\right] .
\end{aligned}
$$

As usual, the eigenvalues are obtained from squaring the Hamiltonian

$$
\begin{aligned}
H^{2} & =\sigma^{a} e_{a}^{i} \hat{p}_{i} e_{b}^{j} \sigma^{b} \hat{p}_{j} \\
& =e_{a}^{i} e_{b}^{j} \sigma^{a} \sigma^{b} \hat{p}_{i} \hat{p}_{j}+e_{a}^{i} \sigma^{a} \sigma^{b}\left\{\hat{p}_{i}, e_{b}^{j}\right\} \hat{p}_{j} \\
& =e_{a}^{i} e_{b}^{j}\left(-\eta^{a b}+i \epsilon^{a b c} \sigma^{c}\right) \hat{p}_{i} \hat{p}_{j}+\frac{i T_{B}^{3}}{2}\left[\sigma^{2}, \sigma^{1}\right] \hat{p}_{z} \\
& =-g^{i j} \hat{p}_{j} \hat{p}_{j}-T_{B}^{3} \sigma_{3} \hat{p}_{z} \\
& =\hat{p}_{y}^{2}+\hat{p}_{z}^{2}+\left(\hat{p}_{x}+T_{B}^{3} \hat{y} \hat{p}_{z}\right)^{2}-T_{B}^{3} \sigma_{3} \hat{p}_{z} .
\end{aligned}
$$

We see (12) is equivalent to a LL problem in a magnetic field [Eq. (A7) for $B^{z}=T_{B}^{3}$ and $q=p_{z}$ in Appendix A]. With those identifications, the spectrum is consequently [from Eq. (A17)]

$$
E\left(p_{z}\right)=\left\{\begin{array}{l} 
\pm \sqrt{p_{z}^{2}+2\left|p_{z} T_{B}^{3}\right| n}, \quad n \geqslant 1 \\
\operatorname{sgn}\left(T_{B}^{3}\right) \chi\left|p_{z}\right|, \quad n=0 .
\end{array}\right.
$$

The lowest Landau level (LLL) is chiral and unpaired, with the momentum-dependent density of states $N_{\mathrm{LL}}\left(p_{z}\right)=\frac{T_{B}^{3}}{4 \pi^{2}}|p|_{z}$ and simple eigenfunctions

$$
\Psi_{\sigma^{3}= \pm 1}\left(y, p_{x}, p_{z}\right) \sim e^{i\left(p_{x} x+p_{z} z\right)} e^{ \pm\left(p_{y} y-p_{z} T_{B}^{3} y^{2} / 2\right)},
$$

where the (pseudo)spin or helicity is determined by $\operatorname{sgn}\left(p_{z} T_{B}\right)$. We stress that the shape of the spectrum is in general also modified due to the momentum replacing the electric charge: left-handed states now disperse as $E<0$ and right-handed states as $E>0$ (or vice versa, depending on the sign of the field) (see Fig. 1).

Analogously to the Landau level calculation with electromagnetic fields, we may turn on a constant torsional electric field parallel to $T_{B}^{3}$ by introducing time dependence to the 


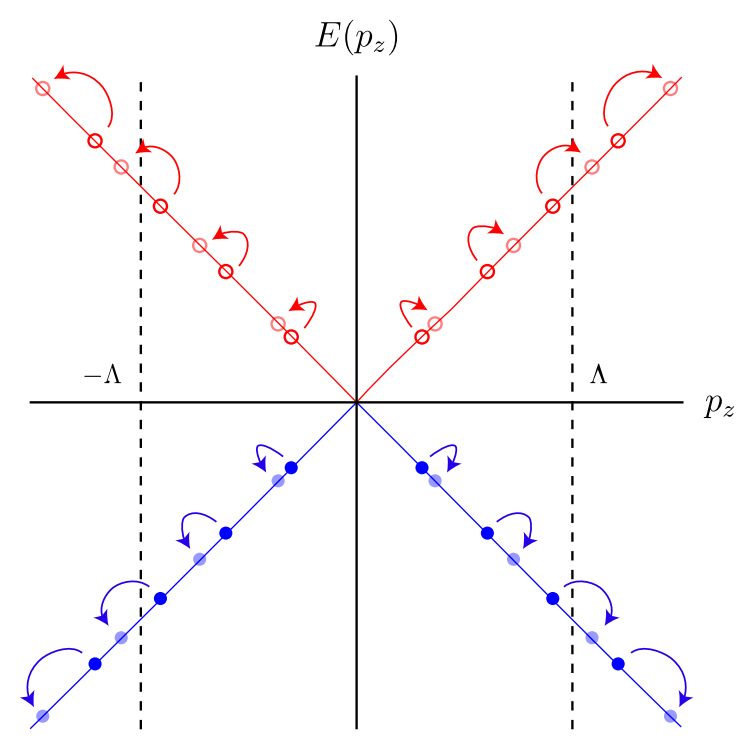

FIG. 2. Relativistic spectral flow at $p_{W}=0$ in the presence of torsion, with the adiabatic transfer of states. Dashed line indicates the location of the cutoff $\Lambda$.

vierbein as $e_{z}^{3}=1+T_{E}^{3} t$ where $T_{E}^{3} t \ll 1$. Then, we have $e_{3}^{z}=\left(1+T_{E}^{3} t\right)^{-1} \approx 1-T_{E}^{3} t$. This induces adiabatic time dependence $\partial_{t} p_{z}=\left(\partial_{t} e_{z}^{3}\right) p_{3}$, analogous to the Lorentz force, which leads to spectral flow of states through the torsional electric field. The number currents, in the vicinity of the node $p_{z}=e_{z}^{3} p_{3}=p_{W z}=0$, are for both chiralities

$$
\begin{aligned}
e j_{\chi}^{0}(t) & =\int_{-\Lambda}^{\Lambda} N_{\mathrm{LL}}\left(p_{z}\right) d p^{3}=\frac{T_{B}^{3}}{2 \pi} \int_{-\Lambda}^{\Lambda} \frac{d p^{3}}{2 \pi}\left|p_{z}\right| \\
& =-\Lambda^{2} \frac{T_{x y}^{3} e_{z}^{3}}{4 \pi^{2}}=-\Lambda^{2} \frac{T_{B}^{3}\left(1+T_{E}^{3} t\right)}{4 \pi^{2}},
\end{aligned}
$$

where a cutoff $\Lambda$ has been introduced to regularize the momentum-dependent density of states integrated over the spectrum. Then, taking into account the fact that the tensorial current density is modified by the volume element $e d^{4} x$ in the presence of torsion (see, e.g., [69,89]), we obtain

$$
\begin{aligned}
e \dot{j}_{\chi}^{0} & =\mp \Lambda^{2} \frac{T_{x y}^{3} \partial_{t} e_{z}^{3}}{4 \pi^{2}}=\mp \Lambda^{2} \frac{T_{B}^{3} T_{E}^{3}}{4 \pi^{2}} \\
& = \pm \frac{\Lambda^{2}}{32 \pi^{2}} \epsilon^{\mu \nu \rho \sigma} T_{\mu \nu}^{3} T_{3 \rho \sigma} .
\end{aligned}
$$

We see that for $E<0$ particles flow below the cutoff, whereas for $E>0$ holes flow above the cutoff, depending on the direction of the torsional electric field (see Fig. 2). This is the vacuum regularization $n_{\mathrm{vac}}=e j_{\mathrm{vac}}^{0}=\sum_{\left|E_{n}\right| \leqslant \Lambda} \operatorname{sgn}\left(E_{n}\right)$ that was also used in Ref. [44], where an additional factor of $\frac{1}{2}$ was present, presumably due to comparison with anomaly inflow from five dimensions. Generalizing this to a fully covariant expression (see Appendix A) gives

$$
\frac{1}{e} \partial_{\mu}\left(e j_{5}^{\mu}\right)=\frac{1}{e} \frac{\Lambda^{2}}{16 \pi^{2}} \epsilon^{\mu \nu \lambda \rho} T_{\rho \sigma}^{a} T_{a \rho \sigma},
$$

and in particular $\partial_{\mu}\left(e j^{\mu}\right)=0$ as required. We discuss the relativistic vacuum and the spectral flow leading to (17), as

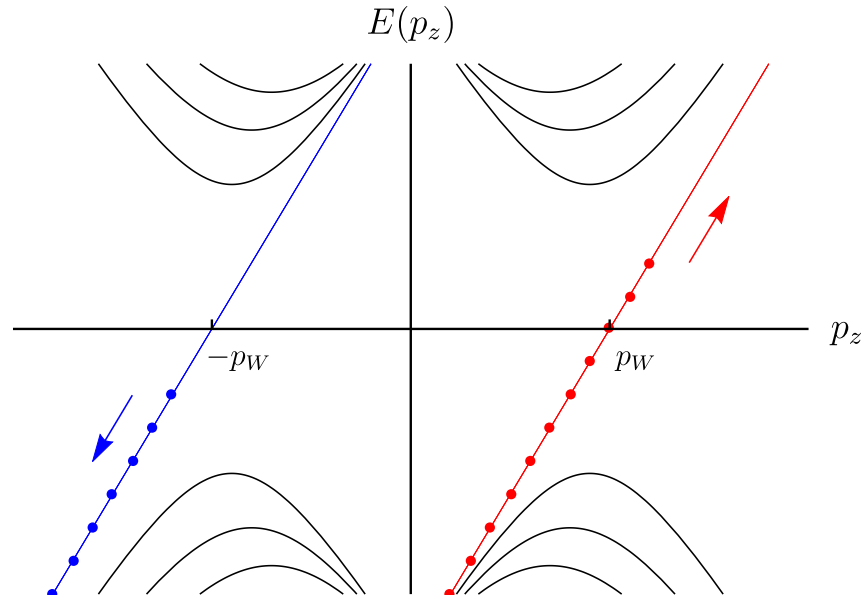

FIG. 3. Left-handed Weyl particles at $p_{z}=p_{W}$ (LLL in red) and right-handed Weyl holes at $p_{z}=-p_{W}$ (LLL in blue) under a torsional magnetic field. Spectral flow is indicated with the arrows.

compared to nodes at finite momenta and axial U(1) fields, more in Sections IIIB and IIIC.

\section{B. Torsional anomaly and spectral flow for $p_{W} \neq 0$}

If we now displace the Weyl nodes in the relativistic case (12) by $p_{z}= \pm p_{W}$ in momentum space, corresponding to a $T$-breaking Weyl system, the spectrum (13) takes the form

$$
E\left(p_{z}\right)=\left\{\begin{array}{l} 
\pm \sqrt{\left(p_{z} \pm p_{W}\right)^{2}+2\left|p_{z} T_{B}^{3}\right| n}, \quad n \geqslant 1 \\
\operatorname{sgn}\left(\chi p_{z} T_{B}^{3}\right)\left(p_{z} \pm p_{W}\right), \quad n=0 .
\end{array}\right.
$$

The lowest, chiral Landau level looks exactly like that of a Weyl fermion in an axial magnetic field [Eq. (A26)]. Higher levels are distorted due to the effective charge carried by the particles being their momentum (see Fig. 3).

Since the node is at finite momentum $p_{W} \neq 0$, also the spectral flow summation (15) is centered around $p_{W} \pm \Lambda^{\prime}$, where $\Lambda^{\prime}$ is a cutoff naturally bounded by the validity of the linear spectrum. For notational convenience and comparison to Eq. (17), we introduce the momentum cutoff as $\Lambda^{\prime}=\frac{\Lambda_{\text {rel }}^{2}}{2} p_{W}$, where we expect $\Lambda_{\text {rel }}^{2} \ll 1$, this being the dimensionless ratio of the cutoff of the linear spectrum to $p_{W}$. This scale is absent in the relativistic case at $p=0$. Repeating the above arguments for the torsional spectral flow results in the expression

$$
\frac{1}{e} \partial_{\mu}\left(e j_{5}^{\mu}\right)=\frac{1}{e} \frac{p_{W}^{2} \Lambda_{\text {rel }}^{2}}{16 \pi^{2}} \epsilon^{\mu \nu \lambda \rho} T_{\mu \nu}^{3} T_{3 \lambda \rho},
$$

which shows that the NY anomaly cutoff is proportional to the node momentum $p_{W}$ [38], and is suppressed by a factor $\Lambda_{\text {rel }}^{2} \ll 1$ corresponding to the validity of the linear Weyl approximation.

\section{Comparison of torsion to U(1) fields}

From Figs. 1-3, we see that the spectrum of torsional LLs resembles the LL spectrum of charged particles in U(1) axial and vector fields, with the effects of the momentum-dependent charge of torsion kept in mind. The contribution of torsion for chiral Weyl fermions can be equivalently cast in terms of 
the axial gauge field $\gamma^{5} S^{\mu} \equiv \gamma^{5} e^{-1} \epsilon^{\mu \nu \lambda \rho} T_{\nu \lambda \rho}$ corresponding to the totally antisymmetric torsion (see, e.g., $[68,69])$. See Appendices A and C about the U(1) case for comparison. We stress that while the spectral equivalence of torsional and U(1) LLs is of course expected, the physical appearance of the anomaly is drastically different: the density of states of the LLs depends on momentum and thus the dimensional coefficient $\Lambda^{2}$ and the need for an explicit UV cutoff appear. Similarly, the physics of Figs. 2 and 3 is completely different, although both arise from spectral flow in momentum space under torsion.

Although the relativistic result in (16) is familiar, there seems to remain confusion in the literature about the role of torsional Landau levels in momentum space and the validity of the NY anomaly due to the explicit UV cutoff. For relativistic Weyl fermions with Lorentz invariance up to arbitrary scales, the spectral flow is symmetric around $p=0$, leading to the conclusion that the anomaly indeed can cancel. This is simply by the observation that, in the absence of Lorentz symmetry breaking at high energy, no net transfer of occupied and empty states in the vacuum takes place during the adiabatic spectral flow (cf. Fig. 2). The net transfer of $j_{5}$ requires left-right asymmetric regularization at the scale of $\Lambda$, with chirality undefined above that scale, while maintaining $\partial_{\mu} j^{\mu}=0$ [44]. At the very least, there is a divergence as $\Lambda \rightarrow \infty$.

In contrast, for quasirelativistic Weyl fermions at finite node momentum and an explicit finite cutoff to the Weyl spectrum, the spectral flow can terminate due to the nonrelativistic corrections at the cutoff scale of $\Lambda^{\prime}$, where also chirality is no longer well defined. This leads to net transport of states and momenta relative to the vacuum (and other quantum numbers of the Weyl fermions, if present). A related fact is that the (sign of) momentum $p_{z}$ plays the role of chirality, which remains physically well defined irrespective of the scale. The flow is composed of particles and antiparticles (holes) at the different nodes.

It would be interesting to study the detailed role of the breakdown of relativistic spectrum and chiral spectral flow numerically, following Ref. [22]. There, only the charge density at finite chemical potential from the node is analyzed, corresponding to Fig. 6, and the expected deterioration away from the Weyl node is verified.

\section{CHIRAL WEYL SUPERFLUIDS AND SUPERCONDUCTORS}

Now, we discuss the role of the torsional anomaly in $p$ wave superfluids and superconductors with gap nodes and associated Weyl-Majorana quasiparticles [13,14,49,73,74,94]. Close to the nodes, the Fermi energy is tuned to the Weyl point due to the existence of the $p+i p$ pairing amplitude. The chiral anomaly is related to the nonconservation of momentum flux $\propto p_{F} e j_{5}^{\mu}$ of the nodal Weyl quasiparticles and the pair condensate $[48,54]$. The relation of this to the torsional gravitational anomaly and the LL spectral flow was pointed out in Ref. [38]. Earlier related work can be found in $[10,12,13,50,51,95,96]$.
The spinless $p+i p$ gap amplitude, with equal spin pairing understood, takes the form

$$
\Delta(\mathbf{p})=\frac{\Delta_{0}}{p_{F}}(\hat{\mathbf{m}}+i \hat{\mathbf{n}}),
$$

where $c_{\perp}=\Delta_{0} / p_{F}$ has units of velocity. The direction $\hat{\mathbf{I}}=$ $\hat{\mathbf{m}} \times \hat{\mathbf{n}}$ is a low-energy Goldstone variable for the condensate. At low-energy, the direction of î can fluctuate and there is combined U(1) gauge symmetry [97] in the $\hat{\mathbf{m}}$-n̂ plane, leading to the Mermin-Ho relations between $\hat{\mathbf{I}}$ and $\mathbf{v}_{s}[3,94,98]$. In the following, we focus on the Landau levels and torsion, keeping the magnitudes of $p_{F}$ and $\Delta_{0}$ fixed. Related to this, for superconductors, the end results apply to the case where the electromagnetic potential $A_{\mu}=0$ which amounts to the gauge where $\mathbf{v}_{s}-\mathbf{A} \rightarrow \mathbf{v}_{s}$. In the following computations, we will set $\mathbf{v}_{s}=0$ as well, since this corresponds to the case where one has only torsion and no spin connection (see Ref. [38] for the general case with superfluid velocity). The orientation of the orthonormal triad $\mathbf{l}$ can still rotate for the torsional textures.

Considering first the homogeneous case, the linearization of the BdG Hamiltonian takes the form of a Weyl Hamiltonian close to the nodes of $E(\mathbf{p})$ at $\mathbf{p}=\mp p_{F} \hat{\mathbf{l}}$ :

$$
\begin{aligned}
H_{\mathrm{BdG}}(\hat{\mathbf{p}}) & =\left(\begin{array}{cc}
\epsilon(\hat{\mathbf{p}}) & \frac{1}{2}\{\hat{\mathbf{p}}, \Delta(\mathbf{p})\} \\
\frac{1}{2}\left\{\hat{\mathbf{p}}, \Delta^{\dagger}(\hat{\mathbf{p}})\right\} & -\epsilon(-\mathbf{p})
\end{array}\right) \\
& \approx \pm \tau^{a} e_{a}^{i}\left(p_{i} \mp p_{F, i}\right) .
\end{aligned}
$$

Note that the BdG excitations are Majorana, $\Phi^{\dagger}(\mathbf{p})=$ $\tau^{1} \Phi(-\mathbf{p})$, as expected in a Bardeen-Cooper-Schrieffer (BCS) paired system. Here we have taken the normal state dispersion $\epsilon(\mathbf{p})=\frac{p^{2}-p_{F}^{2}}{2 m}$, where $m$ is the ${ }^{3} \mathrm{He}$ atom mass. The tetrads are

$$
e_{1}^{i}=c_{\perp} \hat{\mathbf{m}}, \quad e_{2}^{i}=-c_{\perp} \hat{\mathbf{n}}, \quad e_{3}^{i}=-c_{\|} \hat{\mathbf{l}},
$$

where $c_{\|} \equiv \frac{p_{F}}{m}=v_{F}$. Henceforth, to conform with relativistic notation, we will work with dimensionless tetrads in units of $c_{\|}=1$. The dispersion is $E(\mathbf{p})= \pm \sqrt{\epsilon(\mathbf{p})^{2}+|\Delta(\mathbf{p})|^{2}} \approx$ $\pm \sqrt{c_{\|} q_{\|}^{2}+c_{\perp}^{2} q_{\perp}^{2}}$, with $\mathbf{q}=\mathbf{p}-\mathbf{p}_{F}$ for the Weyl quasiparticles. The linear expansion is valid when $\left|\mathbf{p}-\mathbf{p}_{F}\right| \ll p_{F}$, requiring that the remainder

$$
\begin{aligned}
\left.\frac{1}{2} \frac{\partial \epsilon(\mathbf{k})}{\partial k^{i} \partial k^{j}}\right|_{\mathbf{p}_{F}}\left(p-p_{F}\right)^{i}\left(p-p_{F}\right)^{j} & =\frac{1}{2 m}\left(\mathbf{p}-\mathbf{p}_{F}\right)^{2} \\
& \ll e_{a}^{i}\left(\mathbf{p}-\mathbf{p}_{F}\right)_{i} .
\end{aligned}
$$

This leads to the condition, in addition to the trivial $\mid \mathbf{p}-$ $\mathbf{p}_{F} \mid \ll p_{F}$ from the Taylor expansion of $\epsilon(\mathbf{p})$, that

$$
E_{\text {Weyl }} \ll m c_{\perp}^{2}=\left(\frac{c_{\perp}}{c_{\|}}\right)^{2} E_{F},
$$

which will prove important later. In particular, the energy cutoff for the Weyl quasiparticles is anisotropic in momenta $\mathbf{q}=\mathbf{p}-\mathbf{p}_{F}$ around the Weyl point,

$$
q_{\perp} \ll\left(\frac{c_{\perp}}{c_{\|}}\right) p_{F}, \quad q_{\|} \ll\left(\frac{c_{\perp}}{c_{\|}}\right)^{2} p_{F},
$$

in the parallel and perpendicular directions [38]. Here, both are relevant since the three directions are coupled by $\hat{\mathbf{l}}=\hat{\mathbf{m}} \times$ $\hat{\mathbf{n}}$ and the corresponding Mermin-Ho relations. 


\section{A. Landau levels in linear approximation}

To compute the LL levels in the order-parameter texture corresponding to a torsional magnetic field, we can take the "weak-twist" texture $\hat{\mathbf{m}}+i \hat{\mathbf{n}}=\hat{\mathbf{x}}+i \hat{\mathbf{y}}-i T_{B} x \hat{\mathbf{z}}$ with $\left|T_{B} x\right| \ll$ 1 , which corresponds to $\hat{\mathbf{I}}=\hat{\mathbf{z}}+T_{B} x \hat{\mathbf{y}} \approx \hat{\mathbf{z}}[10,50,51]$. The BdG Hamiltonian then takes the form

$$
\begin{aligned}
H_{\mathrm{BdG}} & =\left[\begin{array}{cc}
\epsilon(\hat{\mathbf{p}}) & \frac{1}{2}\left\{\Delta^{i}, \hat{p}_{i}\right\} \\
\frac{1}{2}\left\{\Delta^{\dagger} i, \hat{p}_{i}\right\} & -\epsilon(-\hat{\mathbf{p}})
\end{array}\right] \\
& =\left[\begin{array}{cc}
\epsilon\left(\hat{p}_{x}, p_{y}, p_{z}\right) & \frac{\Delta_{0}}{p_{F}}\left[\hat{p}_{x}+i\left(p_{y}-T_{B} p_{z} x\right)\right] \\
\frac{\Delta_{0}}{p_{F}}\left[\hat{p}_{x}-i\left(p_{y}-T_{B} p_{z} x\right)\right] & -\epsilon\left(-\hat{p}_{x},-p_{y},-p_{z}\right)
\end{array}\right] .
\end{aligned}
$$

Near the gap node $\mathbf{p}_{W}=-p_{F} \hat{\mathbf{1}}$ we may linearize the operator $\epsilon(\hat{\mathbf{p}})$ as $\approx-v_{F} \hat{\mathbf{l}} \cdot\left(\hat{\mathbf{p}}+p_{F} \hat{\mathbf{l}}\right) \approx-v_{F}\left(p_{z}+p_{F}\right)$. This leads to

$$
H_{+}=e_{a}^{i} \tau^{a}\left(p_{i}-p_{F} e_{i}^{3}\right)=\tau^{a}\left(e_{a}^{i} \hat{p}_{i}-p_{F} \delta_{a}^{3}\right)
$$

with

$$
e_{a}^{i}=\left(c_{\perp} \delta_{1}^{i},-c_{\perp}\left[\delta_{2}^{i}-T_{B} x \delta_{3}^{i}\right],-c_{\|} \delta_{3}^{i}\right),
$$

where we remind that $c_{\|} \equiv v_{F}$ and $c_{\perp} \equiv \frac{\Delta_{0}}{p_{F}}$. This corresponds, up to the sign of the field $T_{B}$ and the tetrad, to the case (10) after a rotation in the $\hat{\mathbf{x}}-\hat{\mathbf{y}}$ plane.

In terms of the dimensionless and scaled momenta $p_{a} \equiv e_{a}^{i} p_{i}$, we can define the annihilation operator $\hat{a} \equiv$ $\frac{1}{\sqrt{2\left|T_{B} p_{z}\right|}}\left[\left(\left|T_{B} p_{z}\right| \tilde{x}-p_{\tilde{y}}\right)+i \hat{p}_{\tilde{x}}\right]$ to arrive at the Hamiltonian

$$
H_{p_{z}<0}=\left[\begin{array}{cc}
p_{3}+p_{F} & \sqrt{2\left|T_{B} p_{z}\right|} i \hat{a}^{\dagger} \\
-\sqrt{2\left|T_{B} p_{z}\right|} i \hat{a} & -\left(p_{3}+p_{F}\right)
\end{array}\right],
$$

which is (A7) after $p_{3} \rightarrow p_{3}+p_{F}$. The eigenstates are then

$$
\Psi_{n, p_{z}<0}=\left(\begin{array}{c}
u_{n} \phi_{n} \\
v_{n} \phi_{n-1}
\end{array}\right) e^{i\left(p_{z} z+p_{y} y\right)},
$$

where $\phi_{n} \equiv \phi_{n}(x)$, for $n \geqslant 0$, are harmonic oscillator eigenstates and vanish otherwise. The condition for normalization is $\left|u_{n}\right|^{2}+\left|v_{n}\right|^{2}=1$, corresponding to the $\mathrm{BdG}$ particle and hole amplitudes. We compute $v_{0, p_{z}<0}=0$ [see Eq. (36)]. Carrying out a corresponding calculation at the Weyl point $\mathbf{p}=$ $p_{F} \hat{\mathbf{l}}$, we have the Hamiltonian

$$
H_{p_{z}>0}=\left[\begin{array}{cc}
p_{3}-p_{F} & -\sqrt{2\left|T_{B} p_{z}\right|} i \hat{a} \\
\sqrt{2\left|T_{B} p_{z}\right|} i \hat{a}^{\dagger} & -\left(p_{3}-p_{F}\right)
\end{array}\right],
$$

which can be identified as the left-handed Hamiltonian $H_{-}=$ $-e_{a}^{i} \tau^{a} p_{i}$ after a rotation about $\hat{\mathbf{~}}$ such that $\hat{\mathbf{m}} \rightarrow-\hat{\mathbf{m}}$ and $\hat{\mathbf{n}} \rightarrow$ $-\hat{\mathbf{n}}$. Its eigenstates are

$$
\Psi_{n, p_{z}>0}=\left(\begin{array}{c}
u_{n} \phi_{n-1} \\
v_{n} \phi_{n}
\end{array}\right) e^{i\left(p_{z} z+p_{y} y\right)},
$$

and now $u_{0, p_{z}>0}=0$. Depending on the chirality, i.e., sign of momentum at the node, the LLL is either particlelike or holelike as in Eq. (14). The conclusion is that the spectrum looks like the quasirelativistic spectrum in Fig. 3, when the linear approximation for $\epsilon(\mathbf{p}) \approx \pm c_{\perp}\left(p_{z}-p_{F}\right)$ is valid [Eq. (25)]. This corresponds to the spectrum of axial $\mathrm{U}(1)$ fields with momentum-dependent charge and density of states per LL.
The density of states follows from (A19) in the scaled coordinates, with $e_{\mu}^{0}=\delta_{\mu}^{0}$,

$$
j^{0} d V=e j^{0} d \tilde{V}=\frac{\left|p_{z} T_{B}\right|}{4 \pi^{2}} d \tilde{V} .
$$

\section{B. Anisotropic Newton-Cartan model}

We just showed that the simple order-parameter texture in chiral superfluid or superconductor gives rise to the torsional LLs for the low-energy Weyl quasiparticles, in the linear regime close to nodes. We can, however, consider nonrelativistic quadratic dispersion beyond the linear approximation

$$
\epsilon(\mathbf{p})=\frac{\mathbf{p}^{2}}{2 m}-\mu_{F} \rightarrow \frac{p_{z}^{2}}{2 m}-\mu_{F},
$$

which corresponds to the anisotropic Newton-Cartan spacetime with a (Majorana-Weyl) fermion in Appendix B 2.

The above model has the same regime of validity in the chiral superfluid or superconductor as the linear approximation in Eq. (25) since it also neglects the rotationally invariant dispersion $\epsilon(\mathbf{p})$ of the normal state (see also Ref. [38]). The chiral $p$-wave BCS state and low-energy Weyl description has the uniaxial anisotropy of Eq. (34), however, and this carries to the anomaly and the form of the emergent space-time in Appendix B 2. The other benefit of the anisotropic UV completed model is that the LL spectrum can be computed for momenta far from $p_{F}$, up until $p=0$, corresponding to the filled levels of the nonrelativistic Fermi system, which are absent in the relativistic linear model. This is important for the global properties of the anomaly, including the contribution to the anomalous current from the superfluid vacuum (see Sec. IV C).

The spectrum follows simply from Eqs. (29) and (31) by the substitution $\mp\left(p_{3} \pm p_{F}\right) \rightarrow \pm \epsilon\left( \pm p_{z}\right)$. From squaring the Hamiltonian, the corresponding eigenvalues are at both nodes

$$
\begin{aligned}
& E_{n}= \pm \sqrt{\epsilon\left(p_{z}\right)^{2}+c_{\perp}^{2}\left|T_{B} p_{z}\right| 2 n,} \\
& E_{0}= \pm \operatorname{sgn}\left(p_{z} T_{B}\right) \epsilon\left(p_{z}\right)
\end{aligned}
$$

for $n \geqslant 1$. The LLL state retains the Gaussian form (14). The condition for normalization is $\left|u_{n}\right|^{2}+\left|v_{n}\right|^{2}=1$, and consequently the particle and hole amplitudes are just

$$
u_{n}=\sqrt{\frac{E_{n}+\epsilon\left(p_{z}\right)}{2 E_{n}}}, \quad v_{n}=i \sqrt{\frac{E_{n}-\epsilon\left(p_{z}\right)}{2 E_{n}}} .
$$

With $E_{0}=\epsilon\left(p_{z}\right)$ we have $v_{0}=0$, meaning that the lowestlevel particles appear only for $p_{z}<0$. For $p_{z}>0, u_{0}=0$ when $E_{0}=-\epsilon\left(p_{z}\right)$, so for positive momenta only holes appear at the lowest level, as we found for the linear model. In this case we must, however, remember that the hole spectrum arises due to the Majorana doubling of the BdG spectrum and is not physical. This cancels with a corresponding factor of 2 from spin degeneracy in the Fermi system. This leads to the LL spectrum in Fig. 4.

\section{Spectral flow, anomalous axial density, and vacuum current}

Now we are equipped to compute the spectral flow resulting from torsional Landau levels, corresponding to the 


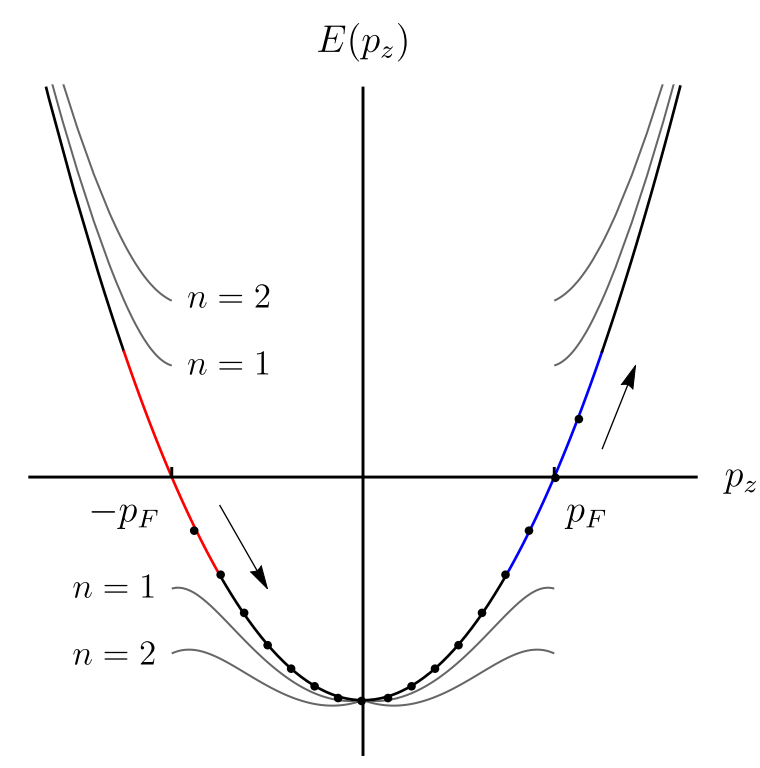

FIG. 4. The torsional LL spectrum for the anisotropic NewtonCartan model in chiral superfluids and conductors with the spectral flow indicated. Note that we have inverted the holelike right-handed Landau level at $-p_{F}$ and the spectrum is particle-hole doubled. Overall, there is a corresponding factor of 2 from spin degeneracy.

covariant torsional NY anomaly. For the anisotropic NewtonCartan model, we can also compute the vacuum current of the condensate since the dispersion takes into account the filled states below the Fermi level, in contrast to the linear model. Interestingly, the resulting anomalous current is the one corresponding to the consistent anomaly $[18,99]$.

\section{Axial density and covariant anomaly}

In chiral $p+i p$ system, the torsional spectral flow leads to the anomalous density corresponding to the covariant anomaly

$$
e j_{ \pm}^{0}=\int_{\mp p_{F}-\frac{p_{F} \Lambda_{\mathrm{rel}}^{2}}{2}}^{\mp p_{F}+\frac{p_{F} \Lambda_{\mathrm{rel}}^{2}}{2}} d p^{3} N_{\mathrm{LL}}\left(p_{z}\right)= \pm \frac{p_{F}^{2}\left(\frac{c_{\perp}}{c_{\|}}\right)^{2}}{4 \pi^{2}} T_{B} e_{z}^{3},
$$

where the cutoff for the anomaly (19) is taken at $\Lambda^{2} / p_{F}^{2}=$ $\Lambda_{\text {rel }}^{2}=\left(\frac{c_{\perp}}{c_{\|}}\right)^{2}$. This cutoff corresponds to Eq. (25) with the condition " $\frac{1}{2} \ll 1$ " in terms of the validity of the Weyl spectrum. Remarkably, although outside the strict validity of the Weyl approximation, the LL results match the more general torsional contribution for the NY anomaly including curvature [38]. This result was found by matching the NY anomaly on emergent space-time to the hydrodynamic linear momentum anomaly of the chiral BCS $p$-wave system. There, inclusion of the effects of superflow leads to a spin connection and curvature perpendicular to $\hat{\mathbf{l}}$, as required by the Mermin-Ho relations [98], producing the leading $p_{F}^{2}$ term in the NY anomaly. The cutoff Eq. (25) in typical ${ }^{3} \mathrm{He}-\mathrm{A}$ liquid is actually $\Lambda_{\text {rel }}^{2}=\frac{c_{\perp}^{2}}{c_{\|}^{2}} \sim 10^{-6}$.

In the chiral superfluid (or superconductor) the above result holds for both the linear quasirelativistic and the anisotropic Newton-Cartan (NC) space-time, as defined by the tetrad (22).
This simply follows from the fact that the cutoff for the validity of both models coincides with (25) since $\hat{\mathbf{i}}=\hat{\mathbf{m}} \times \hat{\mathbf{n}}$, making the triad dependent on all directions $[38,97,98]$. In the LL model we also approximated $\hat{\mathbf{I}} \approx \hat{\mathbf{z}}$ which for the general nontrivial textures is valid up to higher-order corrections [50].

\section{Anomalous axial current and consistent anomaly}

In contradistinction to the anomalous density at the nodes, we can compute the anomalous vacuum (momentum) current for the nonrelativistic anisotropic NC model (34). This corresponds to the anomalous superfluid momentum from the filled states below $p_{F}$ [10]. From the UV completion, the global spectrum is consistent with the nonrelativistic symmetries also outside the vicinity of the Weyl points. The anomalous vacuum momentum current is given by

$$
\mathbf{j}_{\mathrm{anom}, \|}=-2 \int_{0}^{p_{F}} d p^{3} N_{\mathrm{LL}}\left(p_{z}\right) p_{3}=-\frac{p_{F}^{3}}{6 \pi^{2}} \hat{\mathbf{l}}(\hat{\mathbf{l}} \cdot \nabla \times \hat{\mathbf{l}})
$$

and, formally, even extending to $p_{z}=0$, there is no need for a cutoff (see Fig. 4). The current (38) is $p_{F} \hat{\mathbf{I}}$ times the consistent anomaly [99], and arises from the integration over the filled Fermi states $[18,58]$ in contrast to the node density (37).

This is actually the correct hydrodynamic result for the (weak-coupling) BCS system $[10,48,50]$ to lowest order in gradients since the final answer for the anomalous vacuum current is sensitive only to the $e_{3}=\hat{\mathbf{I}}$ direction, even in the presence of $\mathbf{v}_{s}$ (corresponding to curvature in the perpendicular plane). Using superfluid hydrodynamics, the time derivative of (38) produces the covariant anomaly implied by the Weyl nodes $[10,38,48]$. If we assume, without any supporting arguments, that the curvature and torsion contribute to the current (38) as they enter the anomaly (B14), we get the same result if we apply the cutoff (25) as above, even in the linear model. We note that these findings are corroborated by the thermal contribution to the NY anomaly and superfluid free energy in Ref. [81]. In this way, the proper inclusion of curvature in the low-energy theory is expected to ensure that states far away from the Fermi surface do not contribute to the currents.

These considerations are beyond the LL spectral flow argument and we emphasize that the current (38) should be derived from a corresponding Wess-Zumino term, generalized for torsional space-times $[18,70,76,79,100-102]$. See especially [76], where the consistent and covariant anomalies are discussed in an anisotropic Lifshitz model, closely related to Eq. (B13). We leave the study of the consistent vacuum current from the perspective of gravitational anomalies with torsion for the future.

\section{STRAINED WEYL SEMIMETALS}

Semimetals with Weyl fermions arise in solid-state systems where the Fermi energy is tuned to a band crossing in the Brillouin zone $[1,5]$. The tetrads arise universally via the coefficients of the linear expansion of Sec. II. In this case, the electrons are charged and can experience the U(1) anomaly with electromagnetic fields [1,9]. Effective background axial fields from the constant shift of the Weyl node in momentum space lead to the existence of the protected 
Fermi arc states at interfaces with vacuum $[18,19,103]$. Here we would like to analyze and clarify the related, but physically distinct, contribution from elastic strains. In fact, due to the universal coupling of the tetrads to momentum [15,27$29,44]$, as in gravity, one expects that deformations of the lattice geometry lead to effects that probe the Weyl fermions via the background tetrads. The close connections of torsionful geometry and elasticity are discussed in, e.g., [104]. The geometric framework below correctly takes into account the anomalous physics of the momentum-dependent fields (see nevertheless $[17,19,20,59,77,78,84,93,105,106]$ ). Torsional LLs of Sec. III from strain in two dimensions have been recently considered in $[107,108]$.

We start in a roundabout way, first discussing the lowenergy Weyl Hamiltonian and then considering a $T$-breaking lattice model for a realistic material [20].

\section{A. Bloch-Weyl fermions in crystals}

The low-energy Bloch-Weyl Hamiltonian is of the form $[1,5,9]$

$$
\begin{aligned}
h_{ \pm}(\mathbf{k}) & = \pm \sigma^{a}\left(k_{a} \mp k_{W, a}\right)+\text { H.c. } \\
& = \pm \frac{\sigma^{a}}{2} e_{a}^{i}\left(k_{i} \mp k_{W, i}\right)+\text { H.c. }
\end{aligned}
$$

where now

$$
e_{a}^{i}=\left.\frac{\partial H_{a \mathrm{~TB}}(\mathbf{k})}{\partial k_{i}}\right|_{\mathbf{k}_{W}}
$$

are simply the linear coefficients of the expansion of the underlying (tight-binding) Bloch Hamiltonian $H_{\mathrm{TB}}(\mathbf{k})$ near the Weyl nodes. Before we consider lattice deformations and nontrivial tetrads in this model, we remark on the interplay with lattice momentum [15]

$$
\hat{p}_{a}=\frac{i}{2 a} \sum_{\mathbf{x}} c_{\mathbf{x}}^{\dagger} c_{\mathbf{x}+\hat{\mathbf{a}}}-c_{\mathbf{x}+\hat{\mathbf{a}}}^{\dagger} c_{\mathbf{x}}=\sum_{\mathbf{k}} \sin \left(k_{a} a\right) c_{\mathbf{k}}^{\dagger} c_{\mathbf{k}} .
$$

Under nontrivial background fields, the Weyl system itself is anomalous under the lattice translation symmetry $T_{3}=T_{\hat{\mathbf{z}}}$ along the node vector, corresponding to the conservation of the lattice momentum $\hat{p}_{3}$,

$$
T_{\hat{\mathbf{z}}}^{\dagger} c_{ \pm \mathbf{k}} T_{\hat{\mathbf{z}}}=e^{ \pm i a \mathbf{k}_{\mathrm{W}}} c_{ \pm \mathbf{k}_{W}},
$$

since this is an anomalous chiral rotation of the low-energy Weyl fermions at the $T$-breaking nodes $\pm \mathbf{k}_{W}$. Here, $c_{\mathbf{k}}^{\dagger}$ creates the state corresponding to the lattice periodic Bloch state $\left|v_{\mathbf{k}}\right\rangle=\left|v_{\mathbf{k}+\mathbf{K}}\right\rangle$, with wave function

$$
\psi_{\mathbf{k}}(\mathbf{x})=e^{i \mathbf{k} \cdot \mathbf{x}} v_{\mathbf{k}}(\mathbf{x}) .
$$

In the presence of elastic deformations corresponding to torsion, i.e., phonons, the anomalous chiral symmetry corresponding to translations is manifested as the nonconservation of (lattice) node momentum flux $\mathbf{k}_{W} e j_{5}^{\mu}$ between the Weyl fermions and the background phonons [38,47], as found in superfluid ${ }^{3} \mathrm{He}-\mathrm{A}$ for the $(p+i p)$-wave paired Fermi liquid [3]. See also $[16,76,78,109]$.

\section{B. Elastic deformations}

Now we consider general elastic lattice deformations, leading to deformed tetrads $[15,27,28]$. The original unstrained lattice momenta entering the Weyl Hamiltonian are represented as $k_{a}$ and the deformed lattice is given as $k_{i}=e_{i}{ }^{a} k_{a}$ in the coordinate system of the laboratory, where $e_{i}^{a} \neq \delta_{i}^{a}$ to first order in the strains. In particular, this means that Fermi momentum $k_{W, a}$ in the Hamiltonian is held fixed, whereas $k_{W, i}$ with $\delta k_{W, i}=w_{i}^{a} k_{W, a}$ is deformed in the laboratory coordinates. These will couple as expected in the continuum model, if we take into account the finite lattice properly, as we now recall following $[15,28]$. See also $[27,29,78]$. We have the continuum linear strain tensor

$$
\begin{aligned}
& e_{i}^{a}=\delta_{i}^{a}+w_{i}^{a}=\delta_{i}^{a}+\partial_{i} u^{a}, \\
& e_{a}^{i}=\delta_{a}^{i}-w_{a}^{i}=\delta_{a}^{i}-\partial_{j} u^{b} \delta_{a b} \delta^{i j},
\end{aligned}
$$

where $u^{a} / a \ll 1$, in terms of the lattice constant. On the finite lattice, this becomes

$$
\begin{aligned}
& k_{a} \rightarrow k_{a}-w_{a}^{i} \frac{\sin k_{i} a}{a} \approx e_{a}^{i} k_{i}, \\
& k_{i} \rightarrow k_{i}+w_{i}^{a} \frac{\sin k_{a} a}{a} \approx e_{i}^{a} k_{a},
\end{aligned}
$$

where $w_{a}^{i}=\partial_{j} u^{b} \delta_{a b} \delta^{i j}$ is defined above and in the last approximation, the linear approximation for strain as well as $k_{i} a \ll 1$, close to the $\Gamma$ point, are used. In addition we assume that we work with low frequencies corresponding to the acoustic phonons, below the Debye energy [15].

\section{Lattice model}

In general, a model for a $T$-breaking Weyl semimetal consists of layered two-dimensional (2D) Wilson fermions tuned to a zero-energy crossing in three dimensions $[3,22]$. For a model of this kind pertaining to a real material under strain, Ref. [20] considered a "doubled" time-reversal invariant $k \cdot p$ model close to the $\Gamma$ point, with four Weyl nodes in the Brillouin zone, the minimum for a $P$-breaking system. They equated the anomaly from strain with $\mathrm{U}(1)$ gauge fields, in contrast to torsion. For the ease of direct comparison, we will adopt their model with similar notations. While the $k \cdot p$ model is realistic, it is more convenient to work with an an explicit lattice regularization that produces the same low-energy results independent of lattice constant $a$. The full lattice tightbinding model is [20]

$$
H_{\text {lat }}(\mathbf{k})=\epsilon(\mathbf{k})+\left(\begin{array}{ll}
h_{\text {lat }}(\mathbf{k}) & \\
& -h_{\text {lat }}(\mathbf{k})
\end{array}\right),
$$

where we exclusively focus on the time-reversal odd block $h_{\text {latt }}(\mathbf{k})$ of the $T$-invariant model $[3,20,22]$

$$
\begin{aligned}
h_{\text {lat }}(\mathbf{k})= & t_{z}\left(M-\sum_{i=x, y, z} c_{i} \cos k_{i} a\right) \sigma^{3} \\
& +\left(t_{x} \sin k_{x} a\right) \sigma^{1}+\left(t_{y} \sin k_{y} a\right) \sigma^{2} .
\end{aligned}
$$


For $-1<\frac{M-c_{x}-c_{y}}{c_{z}}<1$ the model $h_{\text {lat }}(\mathbf{k})$ has Weyl points at

$$
a \mathbf{k}_{W}= \pm a \mathbf{k}_{F}=\left(0,0, \pm \arccos \frac{M-c_{x}-c_{y}}{c_{z}}\right),
$$

otherwise it is gapped. The dimensionful tetrads are

$$
e_{a}^{i}\left( \pm \mathbf{k}_{F}\right)=a\left(t_{x}, t_{y}, \pm t_{z} c_{z} \sin a k_{F, z}\right) \delta_{a}^{i} .
$$

The inversion symmetry $P$ acts as $h_{\text {lat }}(\mathbf{k}) \rightarrow \sigma^{z} h_{\text {latt }}(-\mathbf{k}) \sigma^{z}$. For simplicity, we set $c_{z}=1, c_{x, y}=c_{\perp}, t_{x, y}=t_{\perp}$ and assume uniaxial symmetry along $\hat{\mathbf{z}}$ in the following. We expect (45) to hold for the Weyl semimetal model (46), originating from the $k \cdot p$ model close to the $\Gamma$ point.

We can moreover ignore the difference of lattice and coordinate indices in the strains, with $u_{i j}=\frac{1}{2}\left(\partial_{i} u_{j}+\partial_{j} u_{i}\right)+$ $O\left(u^{2}\right)$ the symmetric strain tensor. The strain induces the deformation considered in Refs. [16,19,20]:

$$
\begin{aligned}
\delta h_{\mathrm{lat}}(\mathbf{k})= & -t_{z} \beta_{\mathrm{el}} u_{z z} \sigma^{3} \cos a k_{z} \\
& +t_{\perp} \beta_{\mathrm{el}}\left(u_{x z} \sigma^{1}+u_{y z} \sigma^{2}\right) \sin a k_{z}
\end{aligned}
$$

which gives

$$
\delta e_{a}^{i}=a t_{z} \beta_{\mathrm{el}} u_{i i} \delta_{a}^{i} \sin \left(k_{F} a\right)+a t_{\perp} \beta_{\mathrm{el}} \sum_{i^{\prime} \neq i} u_{i i^{\prime}} \delta_{a}^{i^{\prime}} \cos \left(k_{F} a\right),
$$

where $\beta_{\mathrm{el}}$ is the Grünesein parameter. Restricting to a uniaxial strain corresponding to the axis of the Weyl node orientation, with the approximation that $a k_{F} \ll 1$,

$$
\begin{aligned}
e_{a}^{z} & \rightarrow a t_{z}\left(1+\beta_{\mathrm{el}} u_{z z}\right) \delta_{a 3}+a t_{\perp} \sum_{i=x, y} \beta_{\mathrm{el}} u_{z j} \delta_{a}^{j}, \\
\delta e_{3}^{z} & =a t_{z} \beta_{\mathrm{el}} u_{z z}, \quad \delta e_{1}^{z}=a t_{\perp} \beta_{\mathrm{el}} u_{z x}, \quad \delta e_{2}^{z}=a t_{\perp} \beta_{\mathrm{el}} u_{y z} .
\end{aligned}
$$

This has the (dimensionless) inverse tetrad, up to the neglected terms $O\left(u^{2}\right)$ in strains,

$$
e_{i}^{1}=\hat{\mathbf{x}}, \quad e_{i}^{2}=\hat{\mathbf{y}}, \quad e_{i}^{3}=\hat{\mathbf{z}}-\beta_{\mathrm{el}}\left(\left(\frac{t_{z}}{t_{\perp}}\right) u_{z x},\left(\frac{t_{z}}{t_{\perp}}\right) u_{z y}, u_{z z}\right) .
$$

This is what we expected, based on the corresponding universal continuum limit (44) and the lattice substitution (45) coupling to geometry, apart from the (nonuniversal) couplings $\beta_{\mathrm{el}}$, $\left(\frac{t_{z}}{t_{\perp}}\right)$ between the phonons and electrons of the lattice model [15]. Now in the presence of a time-dependent strain vector $e_{z}^{3} \sim u_{z z}$ and torsion $T_{\mu \nu}^{3} \sim \partial_{x} u_{z y}-\partial_{y} u_{z x}$, spectral flow will arise. For, e.g., torsionally twisted and compressed samples along the $\mathbf{z}$ direction [19,20], the Landau level arguments of Secs. III and IV apply for a torsional magnetic field in the "symmetric" gauge and an adiabatic electric field from $u_{z z}(t)$.

\section{Torsional density of states in anomalous transport}

Armed with the geometric background fields corresponding to torsional (magnetic) field, we can consider the anomaly resulting from the chiral rotation (42) in momentum space. For that, we need to analyze the validity of the Weyl approximation and chiral transport in momentum space as in Sec. III.
The linear Weyl model is defined by the expansion

$$
\begin{aligned}
& t_{z}\left(M-\sum_{i=x, y, z} c_{i} \cos k_{i} a\right) \\
& \quad \approx \frac{t_{z} a^{2}}{2}\left[c_{\perp}\left(k_{x}^{2}+k_{y}^{2}\right)+\left(k_{z} \mp k_{F}\right)^{2}\right] \\
& \quad \approx t_{z} a e_{3}^{i}\left(k_{i}-k_{F, i}\right) \\
& \quad=\left(t_{z} a \sin k_{F} a\right) q_{z}
\end{aligned}
$$

which is accurate up to the ignored terms of the remainder in the expansion. Apart from the trivial $q_{z} \ll k_{F} \ll 1 / a$, also

$$
\begin{aligned}
c_{x} \cos q_{x} a+c_{y} \cos q_{y} a & \approx \frac{c_{\perp} a^{2}}{2}\left(q_{x}^{2}+q_{y}^{2}\right) \\
& =\frac{c_{\perp} a^{2}}{2} q_{\perp}^{2} \\
& \ll \frac{t_{x}}{t_{z}} a q_{x}+\frac{t_{y}}{t_{z}} a q_{y} \\
& =\frac{t_{\perp}}{t_{z}} a q_{\perp}
\end{aligned}
$$

leading to the constraint $q_{\perp} \ll \frac{2 t_{\perp}}{c_{\perp} a t_{z}}$, meaning

$$
E_{\text {Weyl }} \ll \frac{t_{\perp}^{2}}{c_{\perp} t_{z}}
$$

for the perpendicular direction. We are working in the units where $-1<M-2 c_{\perp}<1$ and $\cos k_{F} a=M-2 c_{\perp} \approx$ 1. This leads to the anomalous density from magnetic strain, or the chiral densities at the nodes,

$$
\begin{aligned}
n_{ \pm}(\Lambda)=e j_{ \pm}^{0} & =\int_{ \pm k_{F}\left(1 \mp \frac{\Lambda_{\mathrm{rel}}}{2}\right)}^{ \pm k_{F}\left(1 \pm \frac{\Lambda_{\mathrm{rel}}^{2}}{2}\right)} d k^{3} N_{\mathrm{LL}}\left(k_{z}\right) \\
& =\mp \frac{k_{F}^{2} \Lambda_{\mathrm{rel}}^{2}}{4 \pi^{2}} \beta_{\mathrm{el}}^{2}\left(\frac{t_{z}}{t_{\perp}}\right) T_{B} e_{z}^{3} .
\end{aligned}
$$

This leads to the anomaly (19) when $T_{E}=\partial_{t} u_{z z} \neq 0$. Here we have separated the microscopic parameters from the tetrad (53) and $T_{B}$ is the elastic/gravitational torsion $T_{B}=$ $\partial_{x} u_{z y}-\partial_{y} u_{z x}$, etc. In addition to the lattice model parameters, Ref. [20] also estimates that $T_{B} \lesssim \pi / L$ could be sustained, where $L$ is the length of a torsionally twisted semimetal wire.

It is interesting to recall that for the chiral superfluid, while strictly it must be that $\Lambda^{2} / k_{F}^{2}=\Lambda_{\text {rel }}^{2} \ll 1$ since $q_{z} \ll k_{F}$, we found that the cutoff for chiral transport was still parametrically high, implying " $\frac{1}{2} \ll 1$ ” in terms of the validity of the Weyl description. There, however, due to the orthonormal triad, also the perpendicular direction necessarily couples to the transport, with the cutoff Eq. (25) which in real ${ }^{3} \mathrm{He}-\mathrm{A}$ is $\sim 10^{-6} p_{F}$.

For the semimetal, the case where $q_{z} \sim \frac{t_{\perp}}{t_{z} \sin k_{F} a} q_{\perp} \ll k_{F}$ arises when assuming that we isotropically couple to the perpendicular directions for general strain field configurations in the anomaly. Plugging in real parameters, we expect that for, e.g., $\mathrm{Cd}_{3} \mathrm{As}_{2}, t_{\perp} \sim t_{z} \sin k_{F} a$ [20]. This leads to the cutoff of the order of (57). Another option would be to consider the anisotropic Newton-Cartan model in Appendix B with quadratic spectrum $M-2 c_{\perp}-\cos k_{z} a$ along the Weyl node 


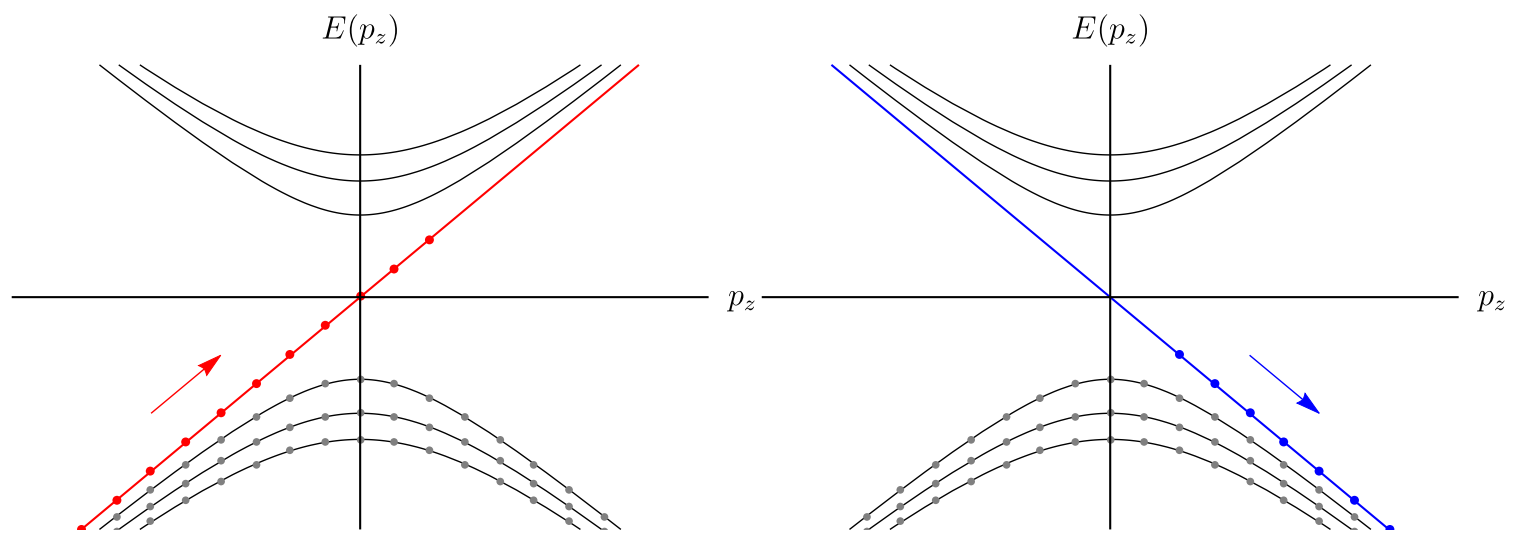

FIG. 5. Dispersion and spectral flow of right-handed (red) and left-handed (blue) particles $(q>0)$ under parallel $\mathbf{B}, \mathbf{E}$.

direction with uniaxial strain only, with the constraint $\Lambda_{\text {rel }}^{2} \ll$ 1 , equivalent to $q_{z} \ll k_{F}$. It is possible that higher orders of $k_{z}$ could be incorporated, following [76]. Here, the same model with different parameters also applies for the Dirac semimetal $\mathrm{Na}_{3} \mathrm{Bi}$ (cf. Ref. [20] and references therein).

Independent of whether one has an axial torsional electric field $\partial_{t} e_{z}^{3} \neq 0$ or an electric field $E^{z}$ driving the spectral flow, as in Figs. 6 and 8, or other chiral effects [43,55,57,58,82] the result is the suppression of the anomalous density proportional to $\Lambda_{\text {rel }}^{2}$, corresponding to the validity of the linear Weyl approximation. We note that this reduction is due to the momentum-dependent density of states in the problem. This, as we have explained, naturally follows from the tetrads and torsion coupling to momenta and should be contrasted with a U(1) (pseudo)gauge field [19,20] and universal density of states proportional to electric charge. Since the lattice model (46) arises by a UV completion designed to match only the low-energy $k \cdot p$ model, and thus is not valid at sufficiently high energies, experiments are needed to fix $\Lambda_{\text {rel }}$ besides the rough order of magnitudes above.

\section{THERMAL EFFECTS}

Finally, we briefly recall and discuss thermal contributions to the torsional anomaly. There are two possible effects: (i) The small but finite temperature enters the NY anomaly as the scale of thermal fluctuations in momentum space $[81,84,85]$. (ii) There is a related finite thermal gradient in the system and one computes the thermal response via Luttinger's fictitious gravitational field [110]. We note that nonzero timelike torsion for the Luttinger space-time implies the non-single-valued time coordinate in the fictitious gravitational field [89]. See also $[90,91,95,111-113]$. Here we focus on the effects of a gravitational field in (ii). Specifically, we assume a thermal gradient

$$
\nabla \sigma=-\frac{1}{T} \nabla T
$$

which is equivalent to a weak gravitational potential $g_{00}=$ $1+2 \sigma$ in the system. The perturbation $\delta g_{00}$ couples to the Hamiltonian (energy current) $H \sim T^{00}$, where $T^{\mu \nu}$ is the energy-momentum tensor. In units where the velocity of propagation is $v_{F}=1$, the metric is

$$
\begin{aligned}
d s^{2} & =e^{+2 \sigma} d t-\delta_{i j} d x^{i} d x^{j} \\
& \approx(1+2 \sigma) d t^{2}-\delta_{i j} d x^{i} d x^{j}
\end{aligned}
$$

from which the linear response to the thermal gradient $\sigma$ can be calculated [110]. This can be generalized to a metric

$$
\begin{aligned}
d s^{2} & =e^{2 \sigma}\left(d t+e^{-\sigma} N_{i} d x^{i}\right)^{2}-\delta_{i j} d x^{i} d x^{j} \\
& =e_{\mu}^{0} e_{\nu}^{0} d x^{\mu} d x^{\nu}-\delta_{i j} d x^{i} d x^{j},
\end{aligned}
$$
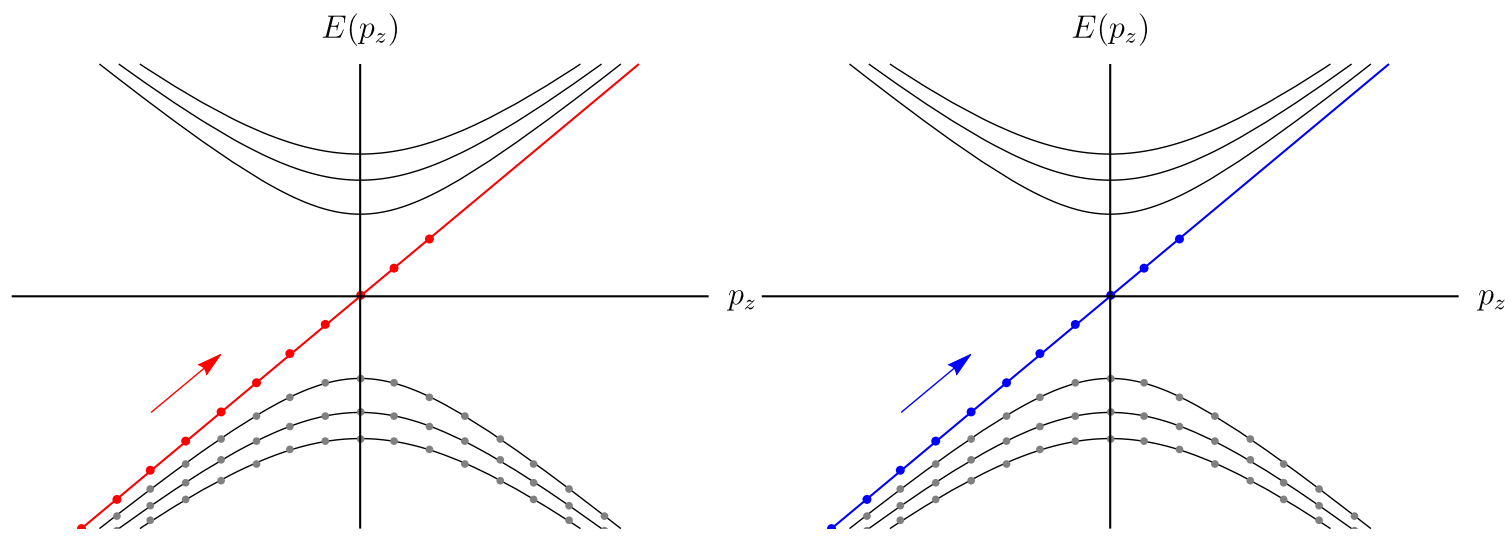

FIG. 6. Spectral flow of parallel $\mathbf{B}_{5}, \mathbf{E}$ with the same conventions as in Fig. 5 . 

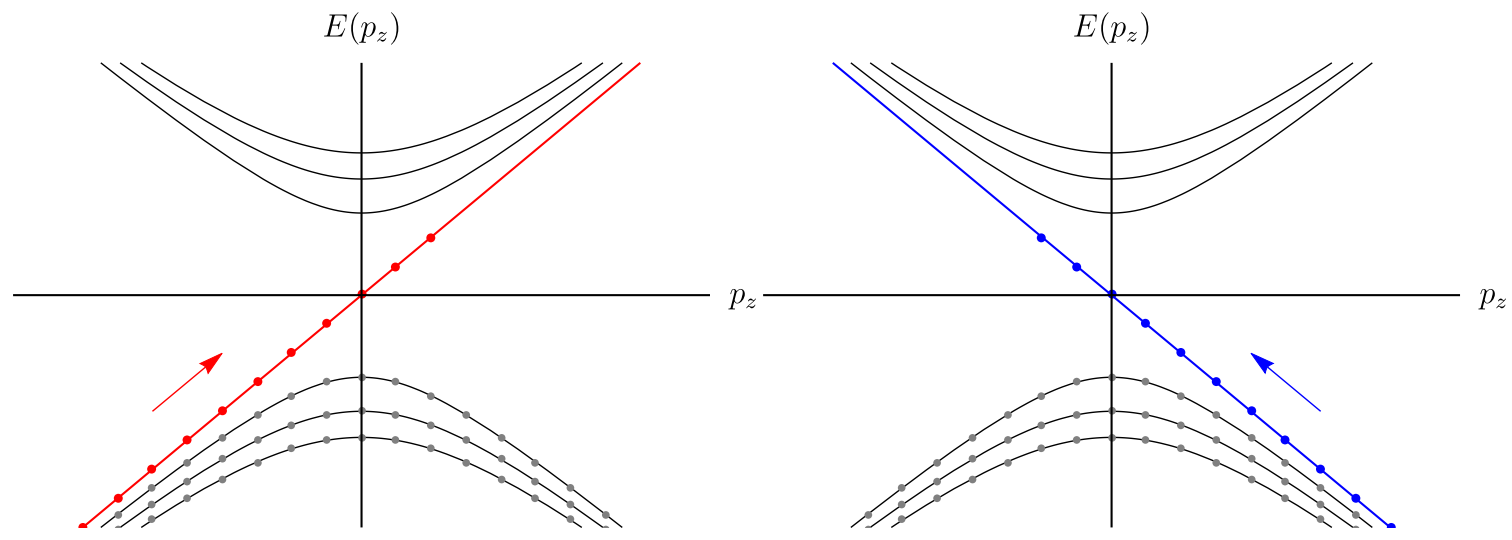

FIG. 7. Spectral flow of parallel $\mathbf{B}, \mathbf{E}_{5}$ with the same conventions as in Fig. 5.

now with a small gravimagnetic potential $[3,112]$

$$
A_{\mu}^{\mathrm{g}}=\left(e^{\sigma}, N_{i}\right) \approx\left(1+\sigma, N_{i}\right) \equiv e_{\mu}^{0},
$$

where $N_{i}$ describes an inverse velocity field in the units where $v_{F}=1$. The gravitational thermal potential is $[3,82,112]$

$$
-\frac{1}{T} \nabla T=\nabla \sigma-\partial_{t} N_{i}
$$

hence,

$$
\begin{aligned}
& e_{\mu}^{0}=\left(e^{\sigma}, N_{i}\right), \quad e_{\mu}^{a}=\delta_{\mu}^{a}, \quad a=1,2,3 \\
& e_{0}^{\mu}=\left(e^{-\sigma}, 0\right), \quad e_{a}^{\mu}=\left(e^{-\sigma} N_{i}, \delta_{a}^{i}\right), \quad a=1,2,3 .
\end{aligned}
$$

In this case Eq. (63) becomes

$$
-\frac{1}{T} \nabla T=\nabla \sigma-\partial_{t} N_{i}=\partial_{i} e_{t}^{0}-\partial_{t} e_{i}^{0}=T_{i t}^{0},
$$

where $T_{\mu \nu}^{0}=\partial_{\mu} e_{\nu}^{0}-\partial_{\nu} e_{\mu}^{0}$ is the temporal torsion, assuming zero temporal spin connection $\omega_{\mu b}^{0} \equiv 0$. It is expected also that one would have possibility for anomalous transport in terms of the combination of thermal gradient and vorticity $T_{i j}^{0}=\partial_{i} N_{j}-\partial_{j} N_{j}$ in the velocity field $N_{i}(x)$, as in the chiral vortical (and magnetic) effect [82,83].

A Weyl node at $p_{W a}=p_{W} \delta_{3 a}$ represents a finite momentum density $\left(P^{i}\right)_{\text {node }}=T^{t i}=p_{W} e_{3}^{i} e j_{5}^{0}$ at low energy [38]. We get momentum density

$$
e T^{t 3}=\frac{p_{W}^{3} \Lambda^{2}}{16 \pi^{2}} \epsilon^{0 \nu \lambda \rho} e_{\nu}^{3} T_{\lambda \rho}^{3}
$$

from the anomalous density $e j_{0}^{5}$ induced by torsion. From the temperature gradient, we similarly expect an energy density of the form

$$
J_{\epsilon}^{t}=e T_{0}^{t}=v_{F} p_{W} e j_{5}^{0}=\frac{p_{W} T^{2}}{12 v_{F}^{2}} \epsilon^{t i j k} e_{i}^{0} T_{j k}^{0},
$$

where $e_{0}^{i} \sim v_{F}$ and $T_{a}^{\mu} \equiv \frac{1}{e} \frac{\delta S}{\delta e_{\mu}^{a}}$ is the (unsymmetrized) energy-momentum tensor. The anomaly of this current would be proportional to $T \nabla T$, and is indeed reminiscent of the chiral vortical effect $[63,82,90,114]$. We can also expect mixed terms, in the sense that there should be a corresponding energy current from both the momentum density and thermal current at the node

$$
J_{\epsilon}^{i}=e T_{0}^{i}=\frac{p_{W} T^{2}}{6 v_{F}^{2}} \epsilon^{0 i j k} e_{j}^{3} T_{0 k}^{0}+\frac{p_{W} T^{2}}{12 v_{F}^{2}} \epsilon^{0 i j k} e_{t}^{0} T_{j k}^{3},
$$

these "mixed" thermal contributions to the anomalous currents were identified and discussed in Ref. [86].

Equation (68) is a "mixed" contribution to the condensed matter torsional anomalies, where the Lorentzinvariant anomaly coefficient $\Lambda^{2} \eta_{a b} \rightarrow \Lambda_{a} \Lambda_{b}$, a generalized
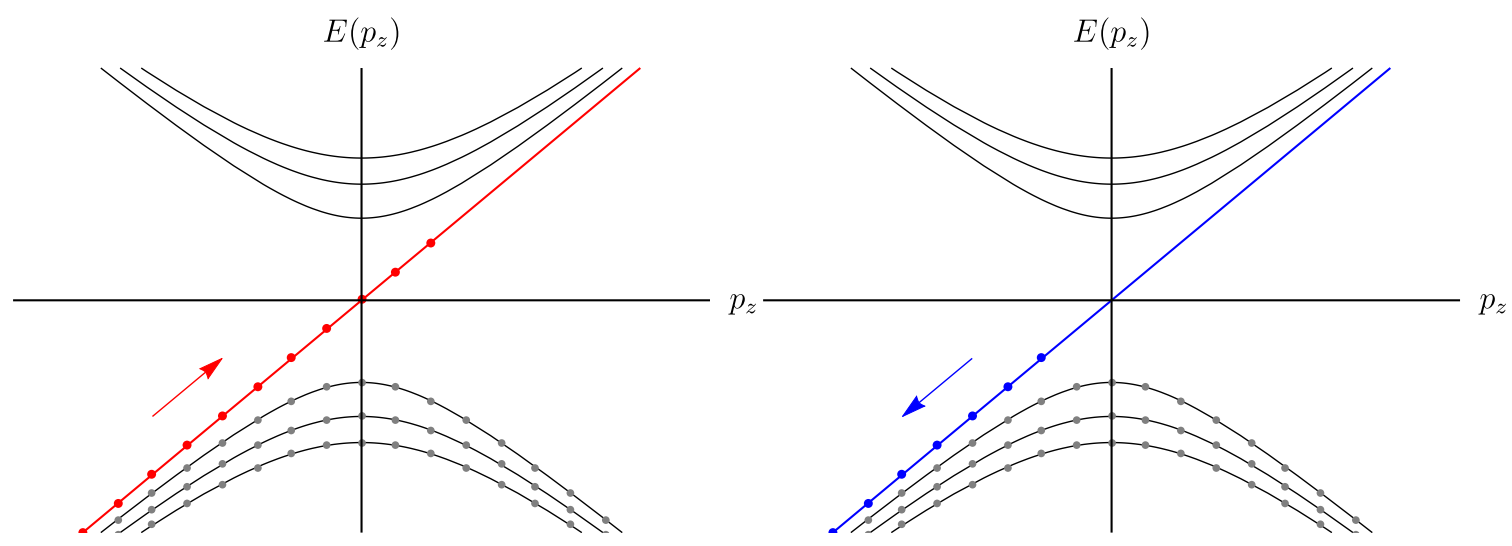

FIG. 8. Spectral flow of parallel $\mathbf{B}_{5}, \mathbf{E}_{5}$ with the same conventions as in Fig. 5. 
anisotropic tensor. We expect such terms in general in various condensed matter systems depending on the symmetries, perturbations, and cutoffs. We leave the detailed discussion of such thermal gravitational contributions for the future; see, however, [84,86] and the general discussion in [81].

\section{CONCLUSIONS AND OUTLOOK}

In this paper, we have argued for the emergence of nonzero torsional anomalies in Weyl (and Dirac) systems with simple Landau level arguments. In particular, we were motivated by the possibility of nonzero torsional Nieh-Yan anomalies in condensed matter systems with an explicit cutoff and the lack of relativistic Lorentz symmetries. For the anomaly, the spectral flow in the presence of torsion clearly renders nonzero results for Weyl nodes at finite momentum. Although obtained with simple field configurations corresponding to Landau levels, they are expected to generalize covariantly to the general gravitational anomaly in terms of the relevant spatial symmetries of the system. Low-energy tetrads and geometry couple to the Weyl fermions in an almost universal way, even in lattice models [15,16], giving our results wide applicability. In Appendix B, we discussed two idealized space-times related to these symmetries, the linear Riemann-Cartan and the anisotropic Newton-Cartan space-time with quadratic dispersion. We find that the symmetries, low-energy dispersion, and $\nabla_{\mu} k_{W \nu}=0$ under deformations constrain the low-energy geometry giving rise to the anomaly (see Appendix B).

More formally, what we did amounts to applying the $K$ theory theorem of Horava [4] to the geometry of specific Weyl nodes in three dimensions, by keeping track of the UV symmetries and scales in the problem for the precise form of the emergent geometry coupling to the quasiparticles. The topology only guarantees the effectively Dirac-type spectrum, with everything else depending on the microscopics.

We also briefly discussed the thermal torsion via Luttinger's fictitious space-time since we can expect mixed anomalies already from the inclusion of thermal gradients. This connects to gravitational anomalies and transport in general [81]. The relation to anomaly coefficients in linear-response thermal transport arising from high-order gravitational anomalies [61,62,80,114-116] should be further explored. From the nonuniversal torsional anomaly the expected gravitational anomaly polynomials at finite temperature arise already at the level of linear response [81]. Moreover, we expect that the emergent tetrads with coordinate dependence arise rather generally in any Weyl system, making sense of evaluating the linear response to these, even in flat space.

We clarified the relation between momentum space pseudogauge fields and the emergent tetrads (cf. Sec. IIIC and Appendices A and C). Importantly, the spectral or Hamiltonian correspondence between the torsional and electromagnetic LL problems is not yet enough for the anomalies to match in general. Remarkably, the simple LL spectral flow argument is enough to identify the nonuniversal UV cutoff $\Lambda$ appearing in the NY anomaly term. More generally, the UV scale appears due to the termination of anomalous chiral transport from such emergent fields and is related to the Weyl point momentum $p_{W}$ and the regime of validity of the effective Weyl-Dirac description, as anticipated in $[68,69]$. For the chiral superfluid or superconductor, the scale $\Lambda$ is essentially fixed by the spontaneous symmetry-breaking pattern $[38,48]$ and matches with experiment [54]. This is similar to two-dimensional (torsional) Hall viscosity [35,117,118] from spontaneous symmetry breaking. On the other hand, for the Weyl semimetal such a scale is absent $[75,76]$ but $\Lambda$ is still proportional to $k_{W}$, although presumably much smaller. Experiments in strain-engineered fermionic Weyl systems are needed.

In the presence of independent $\mathrm{U}(1)$ fields and momentum space tetrads, we should also expect many mixed torsional terms, as studied, e.g., in [44,72,93]. These should also be carefully reconsidered with regards to finite node momentum, where again we expect differences to relativistic fermions. On this note, our results for the anomaly at finite momentum are in contrast to [105], where a relativistic model at $p=0$ with torsion is analyzed with (pseudo)gauge fields without consideration of finite node momentum and the cutoff of the quasirelativistic dispersion.

Many interesting avenues remain in the geometric description of emergent gapless fermions with background fields, including also nodal line systems [37,119]. It would be interesting to study the gravitational anomalies in Weyl and Dirac systems with many nodes, taking fully into account the relevant space-group symmetries and defects [16,42,120-122]. More generally, the appearance of low-energy quasirelativistic fermions with exotic geometric backgrounds within feasible experimental reach is expected to give more insight also to the physics of gravity with torsion, although the symmetries and status of the background fields are dramatically different.

\section{ACKNOWLEDGMENTS}

We thank Z.-M. Huang for correspondence on his work, and O. Golan, T. Ojanen, and P. O. Sukhachov for discussions. Finally, we especially thank G. E. Volovik for discussions, support, and collaborations on related subjects. This work has been supported by the European Research Council (ERC) under the European Union's Horizon 2020 research and innovation programme (Grant Agreement No. 694248).

\section{APPENDIX A: REVIEW OF THE CHIRAL ANOMALY AND THE SPECTRAL FLOW ARGUMENT}

In this Appendix, we compute the U(1) anomaly with LL spectral flow in order to fix notations and provide the LL formulas used in the main text.

\section{Weyl fermions in vector and axial U(1) fields}

The simplest way to argue for the axial anomaly in condensed matter systems is the spectral flow argument, utilizing Landau level spectrum in $3+1$ dimensions [1,10]. The linearized Hamiltonian is of the form

$$
H_{R, L}= \pm \sigma^{i}\left(i \partial_{i}-q A_{i ; R, L}\right),
$$

where $A_{i}$ is some $\mathrm{U}(1)$ gauge field with charge $q$, i.e., the charges in the system are quantized in terms of $q$. Note that we assume that the vector and axial gauge field combinations 
could be both nonzero:

$$
A_{\mu}=\frac{1}{2}\left(A_{R}+A_{L}\right)_{\mu}, \quad A_{5, \mu}=\frac{1}{2}\left(A_{R}-A_{L}\right)_{\mu} .
$$

Under these gauge fields, the chiral fermions always experience the chiral anomaly, where the classical conservation laws corresponding to these fields are broken.

These are summarized by the anomaly equations $[18,21-24]$

$$
\begin{aligned}
& \partial_{\mu} j^{\mu}=\frac{q^{2}}{8 \pi^{2}} \epsilon^{\mu \nu \lambda \rho} F_{\mu \nu} F_{5 \lambda \rho}, \\
& \partial_{\mu} j_{5}^{\mu}=\frac{q^{2}}{16 \pi^{2}} \epsilon^{\mu \nu \lambda \rho}\left(F_{\mu \nu} F_{\lambda \rho}+F_{5 \mu \nu} F_{5 \lambda \rho}\right),
\end{aligned}
$$

where $F_{\mu \nu}=\partial_{\mu} A_{\nu}-\partial_{\nu} A_{\mu}$, etc.

Evidently, if the particles couple to both axial and vector gauge fields, extra currents must be introduced to the system to conserve the total particle number, as required by conservation of charge. This is done by adding Bardeen counterterms to the effective action $[18,99]$

$$
\Gamma\left[A, A_{5}\right] \rightarrow \Gamma\left[A, A_{5}\right]-\int \frac{d^{4} x}{4 \pi^{2}} \epsilon^{\mu \nu \rho \sigma} F_{\mu \nu} A_{\rho} A_{\sigma}^{5},
$$

introducing the countercurrents

$$
\begin{aligned}
& \delta j^{\mu}=\frac{1}{4 \pi^{2}} \epsilon^{\mu \nu \rho \sigma}\left(2 F_{\rho \nu} A_{\sigma}^{5}+F_{\rho \sigma}^{5} A_{\nu}\right), \\
& \delta j_{5}^{\mu}=\frac{1}{4 \pi^{2}} \epsilon^{\mu \nu \rho \sigma} F_{\nu \rho} A_{\sigma} .
\end{aligned}
$$

These modify the axial anomaly in Eq. (A3) to the consistent anomaly in $A_{5}$ (that differs by a factor of $A_{5} \rightarrow \frac{1}{3} A_{5}$ or, equivalently, a Chern-Simons current), so that conservation of $j^{\mu}$ and the vector part of the $j_{5}^{\mu}$ anomaly is maintained:

$$
\begin{aligned}
& \partial_{\mu} j^{\mu}=0, \\
& \partial_{\mu} j_{5}^{\mu}=\frac{q^{2}}{16 \pi^{2}} \epsilon^{\mu \nu \rho \sigma}\left(F_{\mu \nu} F_{\rho \sigma}+\frac{1}{3} F_{\mu \nu}^{5} F_{\rho \sigma}^{5}\right) .
\end{aligned}
$$

Please see Refs. [18,99] and [22-24] for more discussion.

\section{Landau levels}

We consider the minimally coupled Weyl Hamiltonian with vector potential $\mathbf{A}=(-B y, 0,0)$,

$$
\begin{aligned}
H_{\chi} & =\chi \sigma^{i}\left(\hat{p}_{i}-q A_{i}\right) \\
& =\chi\left[\begin{array}{cc}
\hat{p}_{z} & \hat{p}_{x}+q B y-i \hat{p}_{y} \\
\hat{p}_{x}+q B y+i \hat{p}_{y} & -\hat{p}_{z}
\end{array}\right],
\end{aligned}
$$

where $\chi= \pm 1$ denotes the chirality for the fermion. With an eigenstate ansatz $\psi=e^{i\left(p_{z} z+p_{x} x\right)} \phi$ the eigenvalue problem becomes

$$
H_{\chi} \psi=\chi\left[\begin{array}{cc}
p_{z} & p_{x}+q B y-i \hat{p}_{y} \\
p_{x}+q B y+i \hat{p}_{y} & -p_{z}
\end{array}\right] \psi .
$$

For $q B>0$ the off diagonals can be identified as raising and lowering operators for a harmonic oscillator in the $y$ direction (displaced by $p_{x}$ ),

$$
\begin{aligned}
\hat{a} & =\sqrt{2 q B}^{-1}\left[q B y+p_{x}+i \hat{p}_{y}\right], \\
\hat{a}^{\dagger} & =\sqrt{2 q B}^{-1}\left[q B y+p_{x}-i \hat{p}_{y}\right],
\end{aligned}
$$

which satisfy the properties $\left\{\hat{a}, \hat{a}^{\dagger}\right\}=1, \hat{a} \phi_{n}=\sqrt{n} \phi_{n-1}$, and $\hat{a}^{\dagger} \phi_{n}=\sqrt{n+1} \phi_{n+1}$ for eigenstates of the harmonic oscillator $\phi_{n}$. The eigenvalue equation becomes

$$
H_{\chi} \psi=\chi\left[\begin{array}{cc}
p_{z} & \sqrt{2 q B} \hat{a}^{\dagger} \\
\sqrt{2 q B} \hat{a} & -p_{z}
\end{array}\right] \psi .
$$

The energy eigenvalues are obtained from considering the squared Hamiltonian operator:

$$
\begin{aligned}
H_{\chi}^{2} & =(\hat{\mathbf{p}}-q \mathbf{A})^{2}-q \boldsymbol{\sigma} \cdot \mathbf{B} \\
& =\hat{p}_{y}^{2}+p_{z}^{2}+\left(p_{x}+q B y\right)^{2}-q B \sigma_{3},
\end{aligned}
$$

hence,

$$
\begin{gathered}
E^{2}=p_{z}^{2}+2|q B|(n+1)-q B \sigma_{3}, \\
E= \pm \sqrt{p_{z}^{2}+2|q B| n}, \quad n \geqslant 0 .
\end{gathered}
$$

Looking now at the action of the ladder operators on components of the eigenstates $\psi$, they must be of the form

$$
\psi=e^{i\left(p_{z} z+p_{x} x\right)}\left[\begin{array}{c}
\phi_{n} \\
C_{n} \phi_{n-1}
\end{array}\right],
$$

where $\phi_{n}$ are eigenstates of the harmonic oscillator, $\phi_{n-1}=0$, and $C_{n}$ is a factor determined from the eigenvalue equation to be $C_{n}=\frac{\sqrt{2 q B n}}{ \pm E+p_{z}}$ for $n \neq 0$. The $n=0$ state is "half"-occupied since

$$
\psi=e^{i\left(p_{z} z+p_{x} x\right)} \phi_{0}\left[\begin{array}{l}
1 \\
0
\end{array}\right]
$$

with chiral dispersion relation $E=p_{z}$ for $H_{+}$and $E=-p_{z}$ for $H_{-}$, after the elimination of the trivial zero modes $H_{\chi} \Psi=$ 0 .

For $q B<0$ the spectrum is the same but the eigenstates are now

$$
\begin{gathered}
\psi_{n}=e^{i\left(p_{z} z+p_{x} x\right)}\left[\begin{array}{c}
D_{n} \phi_{n-1} \\
\phi_{n}
\end{array}\right], \quad n \geqslant 1 \\
\psi_{0}=e^{i\left(x p_{x}+z p_{z}\right)} \phi_{0}\left[\begin{array}{l}
0 \\
1
\end{array}\right],
\end{gathered}
$$

where $D_{n}=\frac{\sqrt{p_{z} \mp E}}{2|q B| n}$. The zeroth Landau level dispersion relation is $E=-p_{z}$ for $H_{+}$and $E=+p_{z}$ for $H_{-}$.

In summary,

$$
E=\left\{\begin{array}{l} 
\pm \sqrt{p_{z}^{2}+2|q B| n}, \quad n \geqslant 1 \\
\operatorname{sgn}(q B \chi) p_{z}, \quad n=0
\end{array}\right.
$$

The degeneracy of each state can be determined from containing the system within a finite volume $L_{x} L_{y} L_{z}$ and requiring the center of the harmonic oscillator be within it:

$$
0 \leqslant \frac{p_{x}}{|q B|} \leqslant L_{y} .
$$

The $x$ direction is free and is therefore quantized as $p_{x}=n \frac{2 \pi}{L_{x}}$ with $n \in \mathbb{N}$. The $z$ direction is similarly quantized in units of 
$\Delta p_{z}=\frac{2 \pi}{L_{z}}$, so the number of states in the $x y$ plane per $\Delta p_{z}$ is

$$
n=\frac{|q B|}{4 \pi^{2}} L_{x} L_{y} L_{z} .
$$

\section{Spectral flow}

When an electric field parallel to $\mathbf{B}$ is turned on adiabatically, for example, as $\mathbf{A}=\left(-B y, 0,-E_{z} t\right)$, the states flow in the spectrum according to Lorentz's law as $\dot{p}_{z}=q E_{z}$. The unpaired LLL chiral modes flow to specific direction, whereas the higher LLs cancel. The states consequently move in or out of the vacuum depending on their chirality as

$$
\partial_{t} j_{\chi}^{0}=\operatorname{sgn}(q \chi) \frac{q^{2} E B}{4 \pi^{2}}=-\operatorname{sgn}(q \chi) \frac{q^{2}}{32 \pi^{2}} \epsilon^{\mu \nu \rho \sigma} F_{\mu \nu} F_{\rho \sigma} .
$$

We need to generalize (A20) from Minkowski space-time (with metric signature +--- ) to a general space-time with or without torsion. The Landau level calculation generalizes to a nontrivial metric and coordinate-dependent tetrads, when we work in the momentum space $\tilde{p}_{a} \equiv e_{a}^{i} p_{i}, e d \tilde{V}=d V, \tilde{B}=$ $e B$, where $e=\operatorname{det} e_{i}^{a}$, compared to the local Minkowski space. The invariant density of states and fields to be (for $e_{\mu}^{0}=\delta_{\mu}^{0}$ )

$$
\frac{d N}{d V} d V=\frac{|q B|}{4 \pi^{2}} d V=\frac{|q \tilde{B}|}{4 \pi^{2}} d \tilde{V}
$$

which we need to use when we do not want the (scaling of the) tetrads to affect the physical density or flux, the divergence of which we are interested in. With $\dot{p}_{z}=-E_{z}$ the anomaly becomes in coordinate space

$$
\frac{1}{e} \partial_{t}\left(e j_{0}^{\chi}\right)=\frac{1}{e} \frac{\chi q^{2}}{32 \pi^{2}} \epsilon^{\mu \nu \rho \sigma} F_{\mu \nu} F_{\rho \sigma}
$$

which matches (A3) after covariantly generalized to a nontrivial metric.

\section{Inclusion of axial fields}

As discussed, left- and right-handed chiral fermions may also couple independently to different gauge fields $A_{R}$ and $A_{L}$ depending on the chirality:

$$
H_{\chi}=\chi \sigma^{i}\left(p-q A_{R, L}\right)_{i} .
$$

We then define the axial electric and magnetic fields $\mathbf{B}^{5}$ and $\mathbf{E}^{5}$, corresponding to the axial vector potential $A_{5}=\frac{1}{2}\left(A_{R}-\right.$ $\left.A_{L}\right)$. The vector potential is $A=\frac{1}{2}\left(A_{R}+A_{L}\right)$. The corresponding currents are from (A20):

$$
\dot{j^{0}}=\partial_{t} j_{+}^{0}+\partial_{t} j_{-}^{0}=\frac{1}{2 \pi^{2}}\left(E_{z} B_{z}^{5}+E_{z}^{5} B_{z}\right)=\frac{q^{2}}{8 \pi^{2}} \epsilon^{\mu \nu \rho \sigma} F_{\mu \nu} F_{\rho \sigma}^{5},
$$

$$
\begin{aligned}
j_{5}^{0} & =\partial_{t} j_{+}^{0}-\partial_{t} j_{-}^{0} \\
& =\frac{1}{2 \pi^{2}}\left(E_{z} B_{z}+E_{z}^{5} B_{z}^{5}\right) \\
& =\frac{q^{2}}{16 \pi^{2}} \epsilon^{\mu \nu \rho \sigma}\left(F_{\mu \nu} F_{\rho \sigma}+F_{\mu \nu}^{5} F_{\rho \sigma}^{5}\right) .
\end{aligned}
$$

This is the covariant chiral anomaly (A3), represented as spectral flow under parallel electric and magnetic fields. The pictorial version for these equations in form of the LL spectral flow can be found in Figs. 5-8. In a system with both vector and axial fields, the total anomaly must become the consistent anomaly (A6) that maintains charge conservation. This modification affects the coupling of all states to $A_{5}$, including away from the node or requires extra Chern-Simons contributions to the currents.

\section{Weyl node at finite momentum}

In condensed matter systems the Weyl nodes are displaced from $p=0$. Let us fix the symmetry by setting the node at $p_{z}=p_{W}$. It is straightforward to see that shifting the momentum as $\tilde{p}_{i}=p_{i} \pm \delta_{i}^{3} p_{W}$ in the Hamiltonian

$$
H_{ \pm}= \pm \sigma^{i}\left(\tilde{p}_{i}-q A_{i}^{ \pm}\right)
$$

simply shifts the spectrum by $\pm p_{W}$ and the spectral flow in Figs. 5-8.

More importantly, the axial anomaly can then arise from the momentum space structure itself since one has to shift $\Psi$ by $\Psi \rightarrow e^{\mp i \mathbf{p}_{W} \cdot \mathbf{x}} \Psi_{W}$ to obtain the low-energy Weyl excitation $\Psi_{W}$. While this gives almost the same Hamiltonian and spectrum as for of $\mathrm{U}(1)$ fields, the momentum dependence is crucial in the case of the protected Fermi arcs [19], anomalous quantum Hall effect [47,56], as well as the torsional anomaly with the tetrads analyzed in the main text.

\section{APPENDIX B: EMERGENT LOW-ENERGY SPACE-TIMES}

We have discussed how the geometry of the low-energy dispersion affects the anomaly in terms of nonhomogeneous tetrads with torsion in Sec. II. This geometry is dependent on the form of the low-energy dispersion and the nonrelativistic symmetries present in the condensed matter system.

In this section, we describe the full low-energy background geometry corresponding to a nontrivial space-time [35,38]. The original torsional anomaly is derived for relativistic fermions on the background of a torsional Riemann-Cartan space $[65,67]$. We discuss the detailed form of the emergent space-times we should associate to the low-energy geometry in nonrelativistic systems, contrasting Riemann-Cartan and Newton-Cartan space-times. The low-energy geometry in terms of a tetrad and connection can be fixed only by the symmetries in the problem, related to the UV completion.

\section{Quasirelativistic Riemann-Cartan fermions}

We briefly summarize quasirelativistic fermions on curved Riemann-Cartan space-times here (for more see, e.g., $[35,38,44,64,67,68])$. These space-times are defined via an orthonormal frame $e^{a}=e_{\mu}^{a} d x^{\mu}$, giving rise to metric as in (6), and a (matrix) spin connection $\hat{\omega}_{\mu} d x^{\mu}=\omega_{\mu b}^{a} d x^{\mu}$, both of which couple to the Dirac (and Weyl) equation [Eq. (B6) below]. Informally, the $e_{\mu}^{a}$ is a space-time "translation gauge field" which is also the matrix square root of the metric, while $\hat{\omega}$ is the gauge connection corresponding to local (Lorentz) rotations (see, e.g., [65,67]).

As discussed in the Introduction and Sec. II, analogous fields arise in the low-energy Weyl Hamiltonian in condensed matter systems on flat space, giving rise to emergent spacetimes for the low-energy fermions close to the nodes. These 
are, however, not strictly relativistic in the sense that the emergent metric does not follow from locally Lorentz-invariant space-times implied by general relativity, but rather from the low-energy expansion of the microscopic nonrelativistic UV theory. This is what we refer to as quasirelativistic and emergent. Note that the spin connection $\hat{\omega}_{\mu}$ is a gauge field of a local symmetry entering the Dirac operator and its emergence needs a corresponding local symmetry in the condensed matter system. In chiral superconductors and superfluids, a nonzero spin connection is due to the local combined U(1) symmetry, corresponding to gauge and orbital rotations $[35,38,97]$. The tetrad and connection fields give rise to the torsion $T^{a}=d e^{a}+(\hat{\omega} \wedge e)^{a}$ and curvature $\hat{R}=d \hat{\omega}_{\mu}+$ $\hat{\omega} \wedge \hat{\omega}$ field strengths. These tensors equivalently characterize the space-time. From the tetrad one can derive the space-time metric, which enters as a secondary object, in contrast to usual Riemannian space-times where the connection is symmetric in lower indices and uniquely fixed by the metric.

In terms of equations, the basic quantities are the tetrad $e_{\mu}^{a}$ and the connection $\omega_{\mu b}^{a}$. Tensors $X_{b \ldots \nu \ldots}^{a \ldots \ldots}$ can carry local orthonormal (Lorentz) indices and coordinate indices; the two bases can be transformed by contracting with $e_{\mu}^{a}$ or the inverse $e_{a}^{\mu}$. The tetrad is the metric matrix square root

$$
g_{\mu \nu}=e_{\mu}^{a} e_{\nu}^{b} \eta_{a b}, \quad e_{a}^{\mu} e_{b}^{\nu} \eta_{a b}=g^{\mu \nu}
$$

defining a local orthonormal frame in terms of $\eta_{a b}=$ $\operatorname{diag}(1,-1,-1,-1)$. The connection $\nabla$ determines geometric parallel transport in the system as

$$
\nabla_{\mu} X_{v}^{a}=\partial_{\mu} X_{v}^{a}-\Gamma_{\mu \nu}^{\lambda} X_{\lambda}^{a}+\omega_{\mu b}^{a} X_{v}^{b},
$$

etc., extended by linearity and the chain rule. Without loss of generality, the spin connection can be written as

$$
\omega_{\mu b}^{a}=e_{\lambda}^{a} e_{b}^{\nu} \Gamma_{\mu \nu}^{\lambda}-e_{\nu}^{a} \partial_{\mu} e_{b}^{\nu},
$$

where $\Gamma_{\mu \nu}^{\lambda}$ is the coordinate connection with torsion

$$
T_{\mu \nu}^{\lambda}=\Gamma_{\mu \nu}^{\lambda}-\Gamma_{\nu \mu}^{\lambda} .
$$

The metric compatible connection defined by (B3) is consistent with basis changes, as follows from $\nabla e_{\mu}^{a}=0$, and consist of two parts, $\omega_{\mu b}^{a}$ for local orthonormal indices and $\Gamma_{\mu \nu}^{\lambda}$ for coordinate indices. The connection can be decomposed in terms of torsion as

$$
\Gamma_{\mu \nu}^{\lambda}=\stackrel{\circ}{\Gamma}_{\mu \nu}^{\lambda}+C_{\mu \nu}^{\lambda},
$$

where $\stackrel{\circ}{\Gamma}_{\mu \nu}^{\lambda}=\frac{1}{2} g^{\lambda \rho}\left(\partial_{\mu} g_{v \rho}+\partial_{\nu} g_{\mu \rho}-\partial_{\rho} g_{\mu \nu}\right)$ is the torsionfree Christoffel connection fully determined from the metric and $C_{\mu \nu}^{\lambda}=\frac{1}{2}\left(T_{\mu \nu}^{\lambda}+T_{\mu \nu}^{\lambda}-T_{\mu \nu}^{\lambda}\right)$ is the contorsion tensor.

The low-energy quasirelativistic Weyl fermion theory is, in the chiral Dirac fermion basis $\psi=\left(\begin{array}{ll}\psi_{L} & \psi_{R}\end{array}\right)^{T}$, where $\psi_{R, L}$ are Weyl fermions and $\gamma^{a}=\left(\begin{array}{cc}0 & \bar{\sigma}^{a} \\ \sigma^{a} & 0\end{array}\right)$ with $\bar{\sigma}^{a}=\left(1,-\sigma^{i}\right)$,

$$
S_{D}=\int d^{4} x e \frac{1}{2} \bar{\psi} \gamma^{a}\left(e_{a}^{\mu} i D_{\mu}-p_{W a}\right) \psi+\text { H.c., }
$$

where H.c. stands for Hermitian conjugate, $e \equiv \operatorname{det} e_{\mu}^{a}$, and $D_{\mu}$ is the covariant derivative corresponding to the canonical momentum

$$
D_{\mu}=\partial_{\mu}-\frac{i}{4} \omega_{\mu}^{a b} \sigma_{a b}-i q A_{\mu}
$$

where $\gamma^{a b}=\frac{i}{2}\left[\gamma^{a}, \gamma^{b}\right]$ and $A_{\mu}$ is a U(1) gauge potential with charge $q$. They enter the covariant derivative or canonical momentum due to local Lorentz (rotation) and gauge symmetries. For the emergent spin connection to exist, the local rotation symmetry has to be dynamically generated (see Sec. IV and [38]). Importantly to our applications, the quantity $p_{W a}=\left(\mu_{W}, \mathbf{p}_{W}\right)$ is the shift of the of the Weyl (or Dirac) node at chemical potential $\mu_{W}=e_{0}^{v} p_{W v}$ and $\mathbf{p}_{W a}=e_{a}^{i} p_{W i}$ in momentum space. The magnitude of $p_{W a}$ is a UV parameter that is fixed (up to small deformations of $e_{a}^{\mu}$ in coordinate space) in the low-energy theory.

\section{Anisotropic Newton-Cartan fermions}

A related nonrelativistic version of the Riemann-Cartan space-time (B6) is an anisotropic Newton-Cartan (NC) spacetime $[31,32,123,125]$. In the latter, we single out a Newtonian time and, in our case, a preferred spatial direction with quadratic dispersion in contrast to the linear Riemann-Cartan case. In what follows in Secs. IV and V, this preferred direction is along the Weyl node separation, with uniaxial symmetry and anisotropic scaling. Compared to the standard $\mathrm{NC}$ case, there is an additional gauge symmetry corresponding to a $\mathrm{U}(1)$ number conservation and a local Milne boost symmetry along the anisotropy direction $[31,75,76,126]$. These will both be gauge fixed to zero and will be applied mostly in the case of the chiral superconductor and superfluid, where they are absent naturally for Majorana-Weyl fermions. With the time coordinate fixed, the symmetries of the NC space-time then correspond to the generalized Galilean transformations $x^{i} \rightarrow x^{i}+\xi^{i}(x, t)$ [31,32,40,123125], which were utilized in [38] within the linear approximation.

The metric is

$$
g_{\mu \nu}=n_{\mu} n_{\nu}+h_{\mu \nu},
$$

where now $n_{\mu}$ is a spacelike vector, $e_{\mu}^{a}$ a (degenerate) tetrad with metric $h_{\mu \nu}$ restricted to the orthogonal subspace, with $e_{\mu}^{0}=\delta_{\mu}^{0}$ representing Newtonian time,

$$
h_{\mu \nu}=\eta^{a b} e_{\mu}^{a} e_{\nu}^{b}, \quad a, b=0,1,2
$$

with inverses

$$
n_{\mu} \ell^{\mu}=1, \quad e_{\mu}^{a} \ell^{\mu}=0, \quad e_{\mu}^{a} e_{b}^{\mu}=\delta_{b}^{a}, \quad a=0,1,2 .
$$

The connection and torsion follow as [75]

$$
\Gamma_{\mu \nu}^{\lambda}=\stackrel{\circ}{\Gamma}_{\mu \nu}^{\lambda}[h]+\ell^{\lambda} \partial_{\mu} n_{\nu},
$$

from the condition that $\mathcal{L}_{\ell} h_{\mu \nu}=0$, equivalent to $\nabla_{\mu} n_{\nu}=$ $\nabla_{\lambda} h_{\mu \nu}=0$. The torsion is given as

$$
T_{\mu \nu}^{3} \equiv n_{\lambda} T_{\mu \nu}^{\lambda}=-\partial_{\mu} n_{\nu}+\partial_{\nu} n_{\mu}
$$

and the standard spin connection perpendicular to $\ell^{\mu}, \stackrel{\circ}{\omega}_{\mu \nu}[h]$, as in Eq. (B3), amounting to local rotation symmetry along $\ell^{\mu}$. The fact that $n_{\mu}$ is covariantly constant is natural since it can be identified with the direction corresponding to nonzero Weyl node separation in, e.g., $T$-breaking Weyl systems.

We discuss in Sec. IV B the Landau level problem of Majorana-Weyl fermions corresponding to such a space-time, 
with the (right-handed Weyl) action

$$
S_{W}=\int d^{4} x \sqrt{g} \psi^{\dagger}\left[\left(\tau^{a} c_{\perp} e_{a}^{\mu} i D_{\mu}-\tau^{3} \epsilon\left(i \partial_{\ell}\right)\right] \psi+\right.\text { H.c. }
$$

where $\epsilon\left(\partial_{\ell}\right)=\partial_{\ell}^{2} /(2 m)-\mu_{F}$ in the anisotropic direction with $\partial_{\ell}=\ell^{\mu} \partial_{\mu}$, corresponding to the nonrelativistic dispersion and degenerate metric $\ell^{\mu} \ell^{\nu}=g^{\mu \nu}-h^{\mu \nu} . D_{\mu}$ is (B7) with $A_{\mu}=0$ in the perpendicular direction. In this case, the relative anisotropy of the two terms is $c_{\perp} / c_{\|}=m c_{\perp} / p_{F}$, where $p_{F}=$ $\sqrt{2 m \mu_{F}}$ and $c_{\|}=v_{F}$ the Fermi velocity. This NC model can be matched to the results discussed [38]. Note that a very similar model with Lifshitz anisotropy was considered in [75], and the ensuing torsional anomalies for momentum transport in [76]. For a semimetal under strain, the model in Sec. V is correspondingly anisotropic and defines a model for $\mathrm{NC}$ space-time with dispersion $\propto \cos a k_{z}$ but the precise connection to a realistic model with crystal symmetries remains to be worked out in full detail.

\section{Torsional Nieh-Yan anomaly}

We now review the anomaly from torsion $[64,65,67,68]$ and discuss the condensed matter realizations. In general, Weyl fermions coupled to a tetrad and spin connection, representing gravitational fields distinct from the metric $g_{\mu \nu}$, are anomalous in the presence of nonzero torsion and curvature. In the main text the spin connection has been set to vanish for the torsional LL backgrounds. It arises in chiral $p+i p$ systems $[35,38]$ with a local symmetry, while rotational effects are higher order in derivatives in semimetals (see, e.g., [27]).

We focus on a pair of complex fermions of opposite chirality with currents $j_{ \pm}^{\mu}$. The (covariant) torsional anomaly for the axial current $j_{5}^{\mu}=j_{+}^{\mu}-j_{-}^{\mu}$ is [66-70]

$$
\begin{aligned}
\partial_{\mu}\left(e j_{5}^{\mu}\right) & =\frac{\Lambda^{2}}{4 \pi^{2}} \epsilon^{\mu \nu \lambda \rho}\left(\frac{1}{4} T_{\mu \nu}^{a} T_{a \lambda \rho}-\frac{1}{2} e_{\mu}^{a} e_{\nu}^{b} R_{a b \lambda \rho}\right)+O\left(\partial^{4}\right) \\
& =\frac{\Lambda^{2}}{4 \pi^{2}}\left(T^{a} \wedge T_{a}-e^{a} \wedge e^{b} \wedge R_{a b}\right)+O\left(R^{2}\right),
\end{aligned}
$$

where on the second line, we utilize differential form notation $e^{a}=e_{\mu}^{a} d x^{\mu}, \omega_{\mu b}^{a} d x^{\mu}$ and $T^{a}=d e^{a}+\omega_{b}^{a} \wedge e^{b}$ and $R_{b}^{a}=$ $d \omega^{a}{ }_{b}+\omega^{a}{ }_{c} \wedge \omega_{b}^{c}$. For a discussion of the relativistic torsional anomaly term, we refer to $[64,65,67,68,71]$, and for applications in topological condensed matter systems to $[38,44,76-$ $78,84,86]$. For the mixed terms between torsion and $\mathrm{U}(1)$ gauge potentials, see, e.g., [72,93]. We focus on the anomaly contribution solely due to the geometry (tetrads), and we will not consider them. Reference [78] also considered novel "axial" tetrads $e_{\mu R}^{a} \neq e_{\mu L}^{a}$ at two Weyl nodes $R, L$, with (vectorlike) $T^{5}$ appearing as in Eqs. (A2) and (A3). We will require $e_{R}= \pm e_{L}$ but this is actually a rather strong constraint basically only allowing for (improper) rotations that can be gauged away. In the chiral Weyl superfuid or conductor or minimal time-breaking semimetal, $e_{R}=-e_{L}$ but this just the chirality of the nodes and is built in the axial nature of the coupling to torsion. Intriguingly, the trace part of torsion arises as the gauge field of local Weyl scalings but this comes, since nonunitary, with a complex gauge coupling [64]. The presence of different (chiral) tetrad couplings and overall symmetry considerations would be highly interesting for, e.g., parity breaking and other nonminimal Weyl systems with several nodes, some of which can coincide in momentum space.

To conclude, we note the following salient properties related to the NY anomaly term: (i) Despite appearances, it is given by the difference of topological terms, albeit in terms of an embedding in five dimensions [68]. (ii) The NY anomaly term is of second order in gradients and therefore the leading contribution from the background geometry in linear response. (iii) The UV cutoff is isotropic in momentum space by (local) Lorentz invariance but is multiplied by the geometric term, which can be anisotropic. In condensed matter applications, there is no Lorentz invariance, so different anomaly coefficients can arise (see, e.g., Sec. VI). (iv) The total derivative NY term is a Lorentz scalar with contributions from the torsion and curvature, dictated by local exactness $d\left(e^{a} \wedge T_{a}\right)=T^{a} \wedge T_{a}-e^{a} \wedge e^{b} \wedge R_{a b}$. The contributions from torsion and curvature are a priori independent before the geometry (the torsionful connection) is fixed. The anomaly is therefore physical input for the space-time geometry or connection [38]. In more pragmatic terms, the anomaly coefficient $\Lambda^{2}$ can be computed in the case when $\hat{\omega}_{\mu}=0$ from torsional LLs, although the constraints of a consistent space-time geometry should be kept in mind, cf. Eq. (C2).

\section{APPENDIX C: ON THE RELATION OF EMERGENT TORSION AND PSEUDOGAUGE FIELDS}

Here we summarize our findings in relation to earlier literature, where the momentum space field corresponding to the shift of the node is often considered as an axial gauge field $[3,10,16,17,19-21,24,78,93]$. We note that torsion can be shown to enter as an axial gauge field constructed from the totally antisymmetric torsion $\gamma^{5} S^{\mu}=e^{-1} \epsilon^{\mu \nu \lambda \rho} T_{\nu \lambda \rho}[68,69]$ coupling to the momentum. This is essentially what we found in Secs. III and IV with the momentum-space-dependent LL density of states. Still, the LL calculation and anomaly itself should be performed by taking this momentum dependence into account, as we have done in this paper.

How are tetrads with torsion otherwise different from the momentum gauge field? The symmetries corresponding to the tetrads are translations which for finite node momenta, requisite for condensed matter Weyl fermions, correspond to the anomalous chiral symmetry. The geometric formulation with tetrads reveals the background space-time emerging from the node [4]. The overall geometry can be made consistent with the nonrelativistic symmetries away from the Weyl node for a finite momentum range where the low-energy expansion is valid. For the anomalous axial density and anomaly, this leads to the parametric suppression compared to $\mathrm{U}(1)$ anomaly and the UV momentum scale $\Lambda \sim \Lambda_{\text {rel }} p_{W}$. As discussed, the phenomenological implications of this are significant, even without the theoretical recourse to the emergent geometry.

References [15,27,28] for elastic deformations take an explicitly geometrical viewpoint with the strain-induced nonhomogeneous tetrad formalism proposed here. In the simplest possible terms, we start with the Weyl (or Dirac) Hamiltonian 
in flat space with the small deformation $e_{a}^{i}=\delta_{a}^{i}+\delta e_{a}^{i}$ :

$$
\begin{aligned}
H_{+}=\sigma^{a}\left(\hat{k}_{a}-k_{W a}\right) & \rightarrow \frac{\sigma^{a}}{2} e_{a}^{i}\left(\hat{k}_{i}-k_{W i}\right)+\text { H.c. } \\
& =\frac{\sigma^{a}}{2}\left(e_{a}^{i} k_{i}-k_{W a}\right)+\text { H.c. } \\
& \approx \frac{\sigma^{a}}{2}\left(\left[\delta_{a}^{i}+\delta e_{a}^{i}\right] q_{i}+k_{W} \delta e_{a}^{i}\right)+\text { H.c. }
\end{aligned}
$$

where now $k_{W} \delta e_{a}^{i}=-k_{W} \delta e_{i}^{a}$ is the momentum space gauge field in the Hamiltonian with (almost) constant tetrads $[10,15,19,20,27,51,78]$. The right-hand side is the Hamiltonian in coordinate (or laboratory) space, which is the one we have experimental access to, and is deformed with respect to the orthogonal frame of $k_{a}$. We see that the momentum $k_{i}$ couples to $e_{a}^{i}$, as expected, and the shift $k_{W a}$ is constant in the Hamiltonian, irrespective of the deformation. At the same time, the laboratory value is deformed as $k_{W i}=e_{i}^{a} k_{W a}$. In the chiral superfluid and superconductor we explicitly have $p_{W, i}=p_{F} e_{i}^{3}$, giving $p_{F a}=p_{F} \delta_{a}^{3}$. Similarly, for the strained semimetal, the unstrained lattice Fermi wave vector $k_{W a}(x) \rightarrow k_{W a}^{\prime}(x+u) \approx k_{W a}(x)+\partial_{i} u^{a} k_{W a}(x) \equiv e_{i}^{a} k_{W a}$ for the displacement $x^{\prime}=x+u(x)$, giving Eq. (44) as expected.
Reference [78] discusses torsion (and the conservation of momentum) in strained semimetals in terms of a model with both the axial gauge field from the node and the tetrad with elastic deformations [27]. While such a "splitting" between low- and high-energy momenta is in principle allowed, it makes the consideration of the momentum-dependent anomalies more involved. In particular, the momentum anomaly (without EM gauge fields) should be proportional $k_{W} \partial_{\mu}\left(e j_{5}^{\mu}\right)$, as found in [38].

The geometric framework means more generally that we expect

$$
\nabla k_{F a}=0
$$

to hold under the emergent low-energy geometric deformations. This fixes the connection corresponding to the emergent space-time, as discussed in Appendix B and can be taken as the requirements for the consistent assignment of the low-energy geometry for the Weyl fermions. Finally, all the torsional space-times we considered in this paper are gravitoelectromagnetic [3] since the relevant fields can be identified as Abelian, amounting to what was called "minimal coupling" trick in $[15,44]$. Irrespective of the formulation, the gravitational character of the Abelian fields is evident in the (energy-)momentum dependence, including thermal effects.
[1] H. B. Nielsen and M. Ninomiya, The Adler-Bell-Jackiw anomaly and Weyl fermions In a crystal, Phys. Lett. B 130, 389 (1983).

[2] C. G. Callan and J. A. Harvey, Anomalies and fermion zero modes on strings and domain walls, Nucl. Phys. B 250, 427 (1985).

[3] G. E. Volovik, The Universe in a Helium Droplet (Oxford University Press, Oxford, 2003); Exotic properties of superfluid ${ }^{3} \mathrm{He}$ (World Scientific, Singapore, 1992).

[4] P. Hořava, Stability of Fermi Surfaces and $K$ Theory, Phys. Rev. Lett. 95, 016405 (2005).

[5] X. Wan, A. M. Turner, A. Vishwanath, and S. Y. Savrasov, Topological semimetal and Fermi-arc surface states in the electronic structure of pyrochlore iridates, Phys. Rev. B 83, 205101 (2011).

[6] C. Herring, Accidental degeneracy in the energy bands of crystals, Phys. Rev. 52, 365 (1937).

[7] A. A. Abrikosov and S. D. Beneslavskii, Possible Existence Of Substances Intermediate Between Metals And Dielectrics, ZhETF 59, 1280 (1970) [JETP 32, 699 (1971)].

[8] T. O. Wehling, A. M. Black-Schaffer, and A. V. Balatsky, Dirac materials, Adv. Phys. 63, 1 (2014).

[9] N. P. Armitage, E. J. Mele, and Ashvin Vishwanath, Weyl and Dirac semimetals in three-dimensional solids, Rev. Mod. Phys. 90, 015001 (2018).

[10] G. E. Volovik, Normal Fermi liquid in a superfluid ${ }^{3} \mathrm{He}-\mathrm{A}$ at $T=0$ and the anomalous current, Pis'ma ZhETF 42, 294 (1985) [JETP Lett. 42, 363 (1985)].

[11] G. E. Volovik, Chiral anomaly and the law of conservation of momentum in ${ }^{3} \mathrm{He}$-A, Pis'ma ZhETF 43, 428 (1986) [JETP Lett. 43, 551 (1986)].
[12] G. E. Volovik, Analog of gravity in superfluid ${ }^{3} \mathrm{He}-\mathrm{A}$, Pis'ma ZhETF 44, 388 (1986) [JETP Lett. 44, 498 (1986)].

[13] G. E. Volovik, The gravitational topological Chern-Simons term in a film of superfluid ${ }^{3} \mathrm{He}-\mathrm{A}$, Pis'ma ZhETF 51, 111 (1990) [JETP Lett. 51, 125 (1990)].

[14] N. R. Read and D. Green, Paired states of fermions in two dimensions with breaking of parity and time- reversal symmetries and the fractional quantum Hall effect, Phys. Rev. B 61, 10267 (2000).

[15] H. Shapourian, T. L. Hughes, and S. Ryu, Viscoelastic response of topological tight-binding models in two and three dimensions, Phys. Rev. B 92, 165131 (2015).

[16] A. Cortijo, Y. Ferreiros, K. Landsteiner, and M. A. H. Vozmediano, Elastic Gauge Fields in Weyl Semimetals, Phys. Rev. Lett. 115, 177202 (2015).

[17] H. Sumiyoshi and S. Fujimoto, Torsional Chiral Magnetic Effect in a Weyl Semimetal with a Topological Defect, Phys. Rev. Lett. 116, 166601 (2016).

[18] K. Landsteiner, Notes on anomaly induced transport, Acta Phys. Pol. B 47, 2617 (2016).

[19] A. G. Grushin, J. W. F. Venderbos, A. Vishwanath, and R. Ilan, Inhomogeneous Weyl and Dirac Semimetals: Transport in Axial Magnetic Fields and Fermi Arc Surface States from Pseudo-Landau Levels, Phys. Rev. X 6, 041046 (2016).

[20] D. I. Pikulin, A. Chen, and M. Franz, Chiral Anomaly from Strain-Induced Gauge Fields in Dirac and Weyl Semimetals, Phys. Rev. X 6, 041021 (2016).

[21] E. V. Gorbar, V. A. Miransky, I. A. Shovkovy, and P. O. Sukhachov, Consistent Chiral Kinetic Theory in Weyl Materials: Chiral Magnetic Plasmons, Phys. Rev. Lett. 118, 127601 (2017). 
[22] E. V. Gorbar, V. A. Miransky, I. A. Shovkovy, and P. O. Sukhachov, Anomalous transport properties of Dirac and Weyl semimetals, Low Temp. Phys. 44, 487 (2018).

[23] E. V. Gorbar, V. A. Miransky, I. A. Shovkovy, and P. O. Sukhachov, Consistent hydrodynamic theory of chiral electrons in Weyl semimetals, Phys. Rev. B 97, 121105(R) (2018).

[24] R. Ilan, A. G. Grushin, D. I. Pikulin, Pseudo-electromagnetic fields in topological semimetals, Nat. Rev. Phys. 2, 29 (2020).

[25] M. D. Horner, A. Farjami, and J. K. Pachos, Equivalence between vortices, twists, and chiral gauge fields in the Kitaev honeycomb lattice model, Phys. Rev. B 102, 125152 (2020).

[26] J. D. Hannukainen, Y. Ferreiros, A. Cortijo, and J. H. Bardarson, Axial anomaly generation by domain wall motion in Weyl semimetals, Phys. Rev. B 102, 241401(R) (2020).

[27] F. de Juan, M. Sturla, and M. A. H. Vozmediano, Space Dependent Fermi Velocity in Strained Graphene, Phys. Rev. Lett. 108, 227205 (2012).

[28] G. E. Volovik and M. A. Zubkov, Emergent Horava gravity in graphene, Ann. Phys. (NY) 340, 352 (2014); Emergent geometry experienced by fermions in graphene in the presence of dislocations, 356, 255 (2015).

[29] M. A. Zubkov, Emergent gravity and chiral anomaly in Dirac semimetals in the presence of dislocations, Ann. Phys. (NY) 360, 655 (2015); A. Cortijo and M. A. Zubkov, Emergent gravity in the cubic tight-binding model of Weyl semimetal in the presence of elastic deformations, ibid. 366, 45 (2016).

[30] A. Mesaros, D. Sadri, and J. Zaanen, Parallel transport of electrons in graphene parallels gravity, Phys. Rev. B 82, 073405 (2010).

[31] D. T. Son, Newton-Cartan geometry and the quantum Hall effect, arXiv:1306.0638.

[32] R. Banerjee, A. Mitra, and P. Mukherjee, A new formulation of non-relativistic diffeomorphism invariance, Phys. Lett. B 737, 369 (2014); Localization of the Galilean symmetry and dynamical realization of Newton-Cartan geometry, Classical Quantum Gravity 32, 045010 (2015).

[33] J. Nissinen and G. E. Volovik, Type-III and IV interacting Weyl points, JETP Lett. 105, 447 (2017).

[34] A. Westström and T. Ojanen, Designer Curved-Space Geometry for Relativistic Fermions in Weyl Metamaterial, Phys. Rev. X 7, 041026 (2017).

[35] O. Golan and A. Stern, Probing topological superconductors with emergent gravity, Phys. Rev. B 98, 064503 (2018).

[36] L. Liang and T. Ojanen, Curved spacetime theory of inhomogeneous Weyl materials, Phys. Rev. Res. 1, 032006 (2019).

[37] J. Nissinen and G. E. Volovik, Dimensional crossover of effective orbital dynamics in polar distorted ${ }^{3} \mathrm{He}-\mathrm{A}$ : Transitions to antispacetime, Phys. Rev. D 97, 025018 (2018).

[38] J. Nissinen, Emergent Spacetime and Gravitational Nieh-Yan Anomaly in Chiral $p+i p$ Weyl Superfluids and Superconductors, Phys. Rev. Lett. 124, 117002 (2020).

[39] T. Farajollahpour, Z. Faraei, and S. A. Jafari, Solid-state platform for space-time engineering: The 8Pmmn borophene sheet, Phys. Rev. B 99, 235150 (2019); S. A. Jafari, Electric field assisted amplification of magnetic fields in tilted Dirac cone systems, ibid. 100, 045144 (2019); T. Farajollahpour and S. A. Jafari, Synthetic non-Abelian gauge fields and gravitomagnetic effects in tilted Dirac cone systems, Phys. Rev. Res. 2, 023410 (2020).

[40] J. H. Wilson, J. B. Curtis, and V. M. Galitski, Analogue spacetimes from nonrelativistic Goldstone modes in spinor condensates, arXiv:2001.05496.

[41] H. Jia, R.-Y. Zhang, W. Gao, S. Zhang, C. T. Chan, Chiral transport of pseudo-spinors induced by synthetic gravitational field in photonic Weyl metamaterials, arXiv:2009.05954.

[42] V. Juricić, A. Mesaros, R.-J. Slager, and J. Zaanen, Universal Probes of Two-Dimensional Topological Insulators: Dislocation and $\pi$-Flux, Phys. Rev. Lett. 108, 106403 (2012).

[43] Y. You, G. Y. Cho, and T. L. Hughes, Response properties of axion insulators and Weyl semimetals driven by screw dislocations and dynamical axion strings, Phys. Rev. B 94, 085102 (2016).

[44] O. Parrikar, T. L. Hughes, and R. G. Leigh, Torsion, parity-odd response and anomalies in topological states, Phys. Rev. D 90, 105004 (2014); T. L. Hughes, R. G. Leigh, and O. Parrikar, Torsional anomalies, Hall viscosity, and bulk-boundary correspondence in topological states, ibid. 88, 025040 (2013).

[45] A. Farjami, M. D. Horner, C. N. Self, Z. Papić, and J. K. Pachos, Geometric description of the Kitaev honeycomb lattice model, Phys. Rev. B 101, 245116 (2020).

[46] S. L. Adler, Axial-vector vertex in spinor electrodynamics, Phys. Rev. 177, 2426 (1969); J. S. Bell and R. Jackiw, A PCAC puzzle: $\pi^{0} \rightarrow \gamma \gamma$ in the $\sigma$-model, Nuovo Cimento A 60, 47 (1969).

[47] C. Wang, L. Gioia, and A. A. Burkov, Fractional Quantum Hall Effect in Weyl Semimetals, Phys. Rev. Lett. 124, 096603 (2020); M. Thakurathi and A. A. Burkov, Theory of the fractional quantum Hall effect in Weyl semimetals, Phys. Rev. B 101, 235168 (2020).

[48] G. E. Volovik and V. P. Mineev, Orbital angular momentum and orbital dynamics: $3 \mathrm{He}-\mathrm{A}$ and the Bose liquid, ZhETF 81, 989 (1981) [JETP 54, 524 (1981)].

[49] G. E. Volovik, Superfluid properties of ${ }^{3} \mathrm{He}-\mathrm{A}$, Usp. Fiz. Nauk 143, 73 (1984); Superfluid properties of ${ }^{3} \mathrm{He}-\mathrm{A}$, [Sov. Phys.Usp. 27, 363 (1984)].

[50] R. Combescot and T. Dombre, Twisting in superfluid ${ }^{3} \mathrm{He}-\mathrm{A}$ and consequences for hydrodynamics at $T=0$, Phys. Rev. B 33, 79 (1986).

[51] A. V. Balatskii, G. E. Volovik, and A. V. Konyshev, On the chiral anomaly in superfluid ${ }^{3} \mathrm{He}-\mathrm{A}$, ZhETF 90, 2038 (1986) [JETP 63, 1194 (1986)].

[52] G. E. Volovik, Peculiarities in the dynamics of superfluid ${ }^{3} \mathrm{He}-$ A: analog of chiral anomaly and of zero-charge, JETP 65, 1987 (1987).

[53] G. E. Volovik, Orbital angular momentum of vortices and textures due to spectral flow through the gap nodes: example of the ${ }^{3} \mathrm{He}-\mathrm{A}$ continuous vortex, JETP Lett. 61, 958 (1995).

[54] T. D. C. Bevan, A. J. Manninen, J. B. Cook, J. R. Hook, H. E. Hall, T. Vachaspati, and G. E. Volovik, Momentum creation by vortices in ${ }^{3} \mathrm{He}$ as a model of primordial baryogenesis, Nature (London) 387, 689 (1997).

[55] G. Basar, D. E. Kharzeev, and I. Zahed, Chiral and Gravitational Anomalies on Fermi Surfaces, Phys. Rev. Lett. 111, 161601 (2013).

[56] A. A. Zyuzin and A. A. Burkov, Topological response in Weyl semimetals and the chiral anomaly, Phys. Rev. B 86, 115133 (2012).

[57] D. T. Son and N. Yamamoto, Berry Curvature, Triangle Anomalies, and the Chiral Magnetic Effect in Fermi Liquids, Phys. Rev. Lett. 109, 181602 (2012); Kinetic theory with 
Berry curvature from quantum field theories, Phys. Rev. D 87, 085016 (2013).

[58] I. Zahed, Anomalous Chiral Fermi Surfaces, Phys. Rev. Lett. 109, 091603 (2012).

[59] Z. Jian-Hui, J. Hua, N. Qian, and S. Jun-Ren, Topological Invariants of Metals and the Related Physical Effects, Chin. Phys. Lett. 30, 027101 (2013).

[60] D. T. Son and B. Z. Spivak, Chiral anomaly and classical negative magnetoresistance of Weyl metals, Phys. Rev. B 88, 104412 (2013).

[61] K. Landsteiner, Anomalous transport of Weyl fermions in Weyl semimetals, Phys. Rev. B 89, 075124 (2014).

[62] A. Lucas, R. A. Davison, and S. Sachdev, Hydrodynamic theory of thermoelectric transport and negative magnetoresistance in Weyl semimetals, Proc. Natl. Acad. Sci. U. S. A. 113, 9463 (2016).

[63] J. Gooth, A. C. Niemann, T. Meng, A. G. Grushin, K. Landsteiner, B. Gotsmann, F. Menges, M. Schmidt, C. Shekhar, V Süß, et al., Experimental signatures of the mixed axial-gravitational anomaly in the Weyl semimetal $\mathrm{NbP}$, Nature (London) 547, 324 (2017).

[64] H. T. Nieh and M. L. Yan, An identity In Riemann-Cartan geometry, J. Math. Phys. 23, 373 (1982); H. T. Nieh, A torsional topological invariant, Int. J. Mod. Phys. A 22, 5237 (2007).

[65] H. T. Nieh and M. L. Yan, Quantized Dirac field in curved Riemann-Cartan background. I. Symmetry properties, Green's function, Ann. Phys. (NY) 138, 237 (1982).

[66] S. Yajima, Evaluation of the heat kernel in Riemann - Cartan space, Classical Quantum Gravity 13, 2423 (1996).

[67] Y. N. Obukhov, E. W. Mielke, J. Budczies, and F. W. Hehl, On the chiral anomaly in non-Riemannian spacetimes, Found. Phys. 27, 1221 (1997).

[68] O. Chandia and J. Zanelli, Topological invariants, instantons, and the chiral anomaly on spaces with torsion, Phys. Rev. D 55, 7580 (1997); Torsional topological invariants (and their relevance for real life), AIP Conference Proceedings 419, 251 (1998) [arXiv:hep-th/9708138]; O. Chandía and J. Zanelli, Supersymmetric particle in a spacetime with torsion and the index theorem, Phys. Rev. D 58, 045014 (1998).

[69] C. Soo, Adler-Bell-Jackiw anomaly, the Nieh-Yan form, and vacuum polarization, Phys. Rev. D 59, 045006 (1999).

[70] K. Peeters and A. Waldron, Spinors on manifolds with boundary: APS index theorems with torsion, J. High Energy Phys. 02 (1999) 024.

[71] D. Kreimer and E. W. Mielke, "Comment on 'Topological invariants, instantons, and the chiral anomaly on spaces with torsion", Phys. Rev. D 63, 048501 (2001); O. Chandia, J. Zanelli, Reply to Comment on 'Topological invariants, instantons, and the chiral anomaly on spaces with torsion', ibid. 63, 048502 (2001).

[72] S.-I. Kubota, S. Yajima, Y. Higasida, and K. Kawano, Gravitational anomalies with torsion in four dimensions, Classical Quantum Gravity 18, 433 (2001).

[73] G. Palumbo and J. K. Pachos, Holographic correspondence in topological superconductors, Ann. Phys. (NY) 372, 175 (2016)

[74] P. Maraner, J. K. Pachos, and G. Palumbo, Specific heat of 2D interacting Majorana fermions from holography, Sci. Rep. 9, 17308 (2019).

[75] C. Copetti and K. Landsteiner, Anomalous Hall viscosity at the Weyl-semimetal-insulator transition, Phys. Rev. B 99, 195146 (2019).

[76] C. Copetti, Torsion and anomalies in the warped limit of Lifshitz theories, J. High Energy Phys. 01 (2020) 190.

[77] L. Sun and S. Wan, Chiral viscoelastic response in Weyl semimetals, Europhys. Lett. 108, 37007 (2014).

[78] Y. Ferreiros, Y. Kedem, E. J. Bergholtz, and J. H. Bardarson, Mixed Axial-Torsional Anomaly in Weyl Semimetals, Phys. Rev. Lett. 122, 056601 (2019).

[79] Z.-M. Huang, Bo Han, and M. Stone, Hamiltonian approach to the torsional anomalies and its dimensional ladder, Phys. Rev. B 101, 165201 (2020).

[80] K. Landsteiner, E. Megías, and F. Pena-Benitez, Gravitational Anomaly and Transport Phenomena, Phys. Rev. Lett. 107, 021601 (2011).

[81] J. Nissinen and G. E. Volovik, On thermal Nieh-Yan anomaly in Weyl superfluids, Phys. Rev. Res. 2, 033269 (2020).

[82] Z. V. Khaidukov and M. A. Zubkov, Chiral Torsional Effect, JETP Lett. 108, 670 (2018).

[83] S. Imaki and A. Yamamoto, Lattice field theory with torsion, Phys. Rev. D 100, 054509 (2019).

[84] Z.-M. Huang, Bo Han, and M. Stone, The Nieh-Yan anomaly: torsional Landau levels, central charge and anomalous thermal Hall effect, Phys. Rev. B 101, 125201 (2020).

[85] J. Nissinen and G. E. Volovik, On thermal Nieh-Yan anomaly in Weyl systems, JETP Lett. 110, 789 (2019).

[86] L. Liang and T. Ojanen, Topological magnetotorsional effect in Weyl semimetals, Phys. Rev. Res. 2, 022016(R) (2020).

[87] S. Imaki and Z. Qiu, Chiral torsional effect with finite temperature, density and curvature, Phys. Rev. D 102, 016001 (2020).

[88] A. Shitade, Heat transport as torsional responses and Keldysh formalism in a curved spacetime, Prog. Theor. Exp. Phys. 2014, 123I01 (2014).

[89] B. Bradlyn and N. Read, Low-energy effective theory in the bulk for transport in a topological phase, Phys. Rev. B 91, 125303 (2015).

[90] A. Gromov and A. G. Abanov, Thermal Hall effect and geometry with torsion, Phys. Rev. Lett. 114, 016802 (2015).

[91] A. Sekine, Chiral gravitomagnetic effect in topological superconductors and superfluids, Phys. Rev. B 93, 094510 (2016)

[92] E. Witten, Three lectures on topological phases of matter, Riv. Nuovo Cimento 39, 313 (2016);

[93] Z.-M. Huang, L. Li, J. Zhou, and H.-H. Zhang, Torsional response and Liouville anomaly in Weyl semimetals with dislocations, Phys. Rev. B 99, 155152 (2019).

[94] D. Vollhardt and P. Wölfle, The Superfluid Phases of Helium 3 (Taylor \& Francis, Oxford, 1990).

[95] T. Kobayashi, T. Matsushita, T. Mizushima, A. Tsuruta, and S. Fujimoto, Negative Thermal Magnetoresistivity as a Signature of a Chiral Anomaly in Weyl Superconductors, Phys. Rev. Lett. 121, 207002 (2018).

[96] Y. Ishihara, T. Mizushima, A. Tsuruta, and S. Fujimoto, Torsional chiral magnetic effect due to skyrmion textures in a Weyl superfluid ${ }^{3}$ He-A, Phys. Rev. B 99, 024513 (2019).

[97] M. Liu and M. C. Cross, Gauge Wheel of Superfluid ${ }^{3} \mathrm{He}$, Phys. Rev. Lett. 43, 296 (1979).

[98] N. Mermin and T.-L. Ho, Circulation and Angular Momentum in the A Phase of Superfluid Helium-3, Phys. Rev. Lett. 36, 594 (1976).

[99] W. A. Bardeen and B. Zumino, Consistent and covariant 
anomalies in gauge and gravitational theories, Nucl. Phys. B 244, 421 (1984).

[100] G. E. Volovik, Wess-Zumino action for the orbital dynamics of ${ }^{3} \mathrm{He}$-A, Pis'ma ZhETF 44, 144 (1986); Wess-Zumino action for the orbital dynamics of ${ }^{3} \mathrm{He}-\mathrm{A}$ [JETP Lett. 44, 185 (1986)].

[101] A. V. Balatsky, Microscopically derived Wess-Zumino action for ${ }^{3}$ He-A, Phys. Lett. A 123, 27 (1987).

[102] M. Kurkov and D. L. Vassilevich, Gravitational parity anomaly with and without boundaries, J. High Energy Phys. 03 (2018) 072.

[103] F. D. M. Haldane, Attachment of Surface "Fermi Arcs" to the Bulk Fermi Surface: "Fermi-Level Plumbing" in Topological Metals, arXiv:1401.0529.

[104] C. G. Böhmer and Y. Lee, Compatibility conditions of continua using Riemann-Cartan geometry, Math. Mech. Solids, 1081286520961453 (2020).

[105] Z.-M. Huang and B. Han, Torsional Anomalies and Bulk-Dislocation Correspondence in Weyl Systems, arXiv:2003.04853.

[106] K. Landsteiner and Y. Liu, Anomalous transport model with axial magnetic fields, Phys. Lett. B 783, 446 (2018).

[107] É. Lantagne-Hurtubise, X.-X. Zhang, and M. Franz, Dispersive Landau levels and valley currents in strained graphene nanoribbons, Phys. Rev. B 101, 085423 (2020).

[108] M. Oliva-Leyva, J. E. Barrios-Vargas, and G. Gonzalez de la Cruz, Effective magnetic field induced by inhomogeneous Fermi velocity in strained honeycomb structures, Phys. Rev. B 102, 035447 (2020).

[109] J. Nissinen and G. E. Volovik, Tetrads in solids: From elasticity theory to topological quantum Hall systems and Weyl fermions, JETP 127, 948 (2018); Elasticity tetrads, mixed axial-gravitational anomalies, and $(3+1) \mathrm{D}$ quantum Hall effect, Phys. Rev. Res. 1, 023007 (2019).

[110] J. M. Luttinger, Theory of thermal transport coefficients, Phys. Rev. 135, A1505 (1964).

[111] M. Stone, Gravitational anomalies and thermal Hall effect in topological insulators, Phys. Rev. B 85, 184503 (2012).

[112] R. Nakai, S. Ryu, and K. Nomura, Laughlin's argument for the quantized thermal Hall effect, Phys. Rev. B 95, 165405 (2017).

[113] M. N. Chernodub, Alberto Cortijo, and M. A. H. Vozmediano, Generation of a Nernst Current from the Conformal Anomaly in Dirac and Weyl Semimetals, Phys. Rev. Lett. 120,
206601 (2018); V. Arjona, M. N. Chernodub, and M. A. H. Vozmediano, Fingerprints of the conformal anomaly in the thermoelectric transport in Dirac and Weyl semimetals, Phys. Rev. B 99, 235123 (2019).

[114] M. Stone and Y. Kim, Mixed anomalies: Chiral vortical effect and the Sommerfeld expansion, Phys. Rev. D 98, 025012 (2018).

[115] R. Loganayagam and P. Surówka, Anomaly/transport in an ideal Weyl gas, J. High Energy Phys. 04 (2012) 097.

[116] K. Jensen, R. Loganayagam, and A. Yarom, Thermodynamics, gravitational anomalies and cones, J. High Energy Phys. 02 (2013) 088.

[117] N. Read, Non-Abelian adiabatic statistics and Hall viscosity in quantum Hall states and $p_{x}+i p_{y}$ paired superfluids, Phys. Rev. B 79, 045308 (2009).

[118] O. Golan, C. Hoyos, and S. Moroz, Boundary central charge from bulk odd viscosity: Chiral superfluids, Phys. Rev. B 100, 104512 (2019); F. Rose, O. Golan, and S. Moroz, Hall viscosity and conductivity of two-dimensional chiral superconductors, SciPost Phys. 9, 006 (2020).

[119] T. Matsushita, S. Fujimoto, and A. P. Schnyder, Topological piezoelectric effect and parity anomaly in nodal line semimetals, Phys. Rev. Research 2, 043311 (2020).

[120] J. L. Manes, Existence of bulk chiral fermions and crystal symmetry, Phys. Rev. B 85, 155118 (2012).

[121] R. J. Slager, A. Mesaros, V. Juricić, and J. Zaanen, The space group classification of topological band-insulators, Nat. Phys. 9, 98 (2013).

[122] P. Rao and B. Bradlyn, Hall Viscosity in Quantum Systems with Discrete Symmetry: Point Group and Lattice Anisotropy, Phys. Rev. X 10, 021005 (2020).

[123] M. H. Christensen, J. Hartong, N. A. Obers, and B. Rollier, Torsional Newton-Cartan geometry and Lifshitz holography, Phys. Rev. D 89, 061901(R) (2014).

[124] E. A. Bergshoeff, J. Hartong, and J. Rosseel, Torsional Newton-Cartan geometry and the Schrödinger algebra, Class. Quantum Grav. 32, 135017 (2015).

[125] C. Duval, G. Burdet, H. P. Künzle, and M. Perrin, Bargmann structures and Newton-Cartan theory, Phys. Rev. D 31, 1841 (1985).

[126] R. Banerjee and P. Mukherjee, Milne Boost from Galilean gauge theory, Phys. Lett. B 778, 303 (2018). 